\title{
Logarithmic Relaxation in a Colloidal System
}

\author{
M. Sperl \\ Physik-Department, Technische Universität München, 85747 Garching, Germany
}

(Dated: November 17, 2018)

\begin{abstract}
The slow dynamics for a colloidal suspension of particles interacting with a hard-core repulsion complemented by a short-ranged attraction is discussed within the frame of mode-coupling theory for ideal glass transitions for parameter points near a higher-order glass-transition singularity. The solutions of the equations of motion for the density correlation functions are solved for the square-well system in quantitative detail by asymptotic expansion using the distance of the three control parameters packing fraction, attraction strength and attraction range from their critical values as small parameters. For given wave vectors, distinguished surfaces in parameter space are identified where the next-to-leading order contributions for the expansion vanish so that the decay functions exhibit a logarithmic decay over large time intervals. For both coherent and tagged particle dynamics the leading-order logarithmic decay is accessible in the liquid regime for wave vectors of several times the principal peak in the structure factor. The logarithmic decay in the correlation function is manifested in the mean-squared displacement as a subdiffusive power law with an exponent varying sensitively with the control parameters. Shifting parameters through the distinguished surfaces, the correlation functions and the logarithm of the mean-squared displacement considered as functions of the logarithm of the time exhibit a crossover from concave to convex behavior, and a similar scenario is obtained when varying the wave vector.
\end{abstract}

PACS numbers: 61.20.Lc, 82.70.Dd, 64.70.Pf

\section{INTRODUCTION}

The dynamics in an interacting many particle system is conveniently described by density autocorrelation functions $\phi_{q}(t)$ for time $t$ and wave vector $q$. These correlation functions can be measured in both experiment and computer simulation [1]. Mode-coupling theory for ideal glass transitions (MCT) discusses the transition from a liquid to a glass as a bifurcation in the long-time limit of the correlator $\phi_{q}(t)$ [2]. In the liquid state, the correlation function decays to zero. If a control parameter, say density, exceeds some critical value, the longtime limit changes discontinuously from zero to a finite value, a glass transition occurs [3]. This liquid-glass transition is identified with an $A_{2}$ - or fold singularity [4] in the equations of motion of MCT. The simplest example for a liquid-glass transition is found in the hard-sphere system (HSS), where the interaction potential among the particles is zero unless their mutual separation becomes smaller than their diameter where the potential becomes infinitely repulsive, thus preventing the particles from overlapping. The HSS is the system MCT was applied to first [3], and it is also the system for which the most detailed predictions have been worked out [5, 6]. Close to the singularity, the equations of motion can be expanded in asymptotic series. This yields a two-step decay with two related power laws for the short-time and the long-time decay at a liquid-glass transition [2]. The HSS can be realized in colloidal suspensions [7]. Experiments in these systems lead to the conclusion that MCT is able to describe the main aspects of the glass transition qualitatively and some aspects even quantitatively [8].

MCT can also exhibit other singularities than the fold [2]. These higher-order singularities were predicted recently to occur for colloidal systems with short-ranged attraction where $A_{3}$ - and $A_{4}$-singularities were found that are also called cusp and swallowtail [9, 10, 11]. In these systems, the hard-core repulsion is supplemented by a short ranged attraction, e.g. in the square-well system (SWS). A cusp singularity is the endpoint of a line of glass-glass transitions that arises if two different mechanisms of arrest are of the same importance. In the SWS the first mechanism is the hard core repulsion that causes a transition as in the HSS via the well known cage effect. The second mechanism leading to arrest is bond formation introduced by the attractive part of the potential. This latter transition was proposed as relevant for the transition to a gel [10]. If the difference in the two mechanisms is less pronounced, the glass-glass transitions vanish and give rise to an $A_{4}$-singularity. In the SWS this happens as the range of the attraction is increased [11]. The range of attraction considered here is of order less than $20 \%$ of the particle diameter and the strength is about several $k_{\mathrm{B}} T$. This is accessible in colloidpolymer mixtures with nonadsorbing polymer which is well under control experimentally [12]. Higher-order singularities have also been identified for a number of short-ranged potentials with shapes differring from the SWS yielding certain quantitative trends but no qualitative changes [13].

In addition to the success of MCT for the description of the HSS, two findings support the use of this theory for the description of colloids with attraction. First, a reentry phenomenon was predicted by the theory where a glass state is melted upon increasing the attraction [9, 11]. This was subsequently found in several experiments [14, 15] and computer simulation studies [15, 16, 17, 18]. Second, there are indications of logarithmic decay [19] and related anomalous decays [17, 20] that are consistent with scenarios found within MCT [9, 11]. To investigate the dynamics in such systems, apart from computer simulation dynamic light scattering has already been used to determine correlation functions $[14,15,20]$. Direct imaging techniques are available to determine also the mean-squared displacement (MSD) with high precision [21, 22, 23]. The purpose of the present paper is the application of the general theory for higher-order singularities [24] to the SWS and the derivation of testable quanti- 
tative predictions for the correlation functions and the MSD. Certain scenarios have been discussed before for schematic models [24]. Some of these scenarios shall be identified also in the microscopic model in the following.

The paper is organized as follows. In Sec. Ithe equations of motion and the asymptotic solution for the logarithmic decay are summarized and the subdiffusive power law for the MSD is derived. The theory is applied to the $A_{4}$-singularity in the SWS for the correlation functions in Sec. III and for the MSD in Sec. IV Changes in the scenarios when moving from the $A_{4}$-singularity to $A_{3}$-singularities are discussed in Sec. D and Sec. $\mathrm{VI}$ contains a comparison to results obtained for the hard-core Yukawa system (HCY). Section VII presents a conclusion.

\section{ASYMPTOTIC SOLUTIONS}

We shall consider a system of $N$ particles with diameter $d$ in a volume $V$ interacting with a spherical potential. When at time $t$ the $j$ th particle is located at $\vec{r}_{j}(t)$ the density variables are defined as $\rho_{q}(t)=\sum_{j} \exp \left[i \vec{q} \vec{r}_{j}(t)\right]$.

\section{A. Equations of Motion}

The equations of motion for the normalized density correlators $\phi_{q}(t)=\left\langle\rho_{\vec{q}}^{*}(t) \rho_{\vec{q}}\right\rangle /\left\langle\left|\rho_{\vec{q}}\right|^{2}\right\rangle$ within MCT, when Brownian dynamics for the motion in colloids is assumed, are given by [2, 3, 25, 26],

$$
\tau_{q} \partial_{t} \phi_{q}(t)+\phi_{q}(t)+\int_{0}^{t} m_{q}\left(t-t^{\prime}\right) \partial_{t^{\prime}} \phi_{q}\left(t^{\prime}\right) d t^{\prime}=0 .
$$

The initial conditions are $\phi_{q}(0)=1$. The microscopic time scale reads $\tau_{q}=S_{q} /\left(D_{0} q^{2}\right)$. It is given by the short-time diffusion coefficient, $D_{0}$, characterizing the Brownian motion and the static structure factor $S_{q}=\left\langle\left|\rho_{\vec{q}}\right|^{2}\right\rangle$. The mode-coupling approximation results in expressing the kernels $m_{q}(t)$ in terms of the correlators $\phi_{q}(t)$ [2],

$$
m_{q}(t)=\mathcal{F}_{q}\left[\mathbf{V}, \phi_{k}(t)\right] .
$$

As a consequence of the factorization into pair modes for the structural relaxation in simple liquids, $\mathcal{F}_{q}$ is a bilinear functional of the density correlators [3],

$$
\mathcal{F}_{q}[\tilde{f}]=\frac{1}{2} \int \frac{d^{3} k}{(2 \pi)^{3}} V_{\vec{q}, \vec{k}} \tilde{f}_{k} \tilde{f}_{|\vec{q}-\vec{k}|},
$$

and the vertex is determined completely by the static structure of the liquid system [27, 28],

$$
V_{\vec{q}, \vec{k}}=S_{q} S_{k} S_{|\vec{q}-\vec{k}|} \rho\left[\vec{q} \cdot \vec{k} c_{k}+\vec{q} \cdot(\vec{q}-\vec{k}) c_{|\vec{q}-\vec{k}|}\right]^{2} / q^{4} .
$$

The number density is given by $\rho=N / V$ and $c_{q}$ denotes the direct correlation function which is related to the static structure factor $S_{q}$ in the Ornstein-Zernike relation, $S_{q}=1 /[1-$ $\left.\rho c_{q}\right]$, both depend on external control parameters like density or temperature [29]. For the SWS with hard-core diameter $d$, depth of the potential $u_{0}$, and range of the potential $\Delta$, we get three dimensionless control parameters, the packing fraction $\varphi=d^{3} \rho \pi / 6$, the attraction strength $\Gamma=u_{0} /\left(k_{\mathrm{B}} T\right)$ and the relative well width $\delta=\Delta / d$. These can be combined to a control-parameter vector $\mathbf{V}=(\varphi, \Gamma, \delta)$.

It is the long-time limit of the correlation function, $\lim _{t \rightarrow \infty} \phi_{q}(t)=f_{q}$, that determines whether a system is in the liquid regime, where $f_{q}=0$, or in an arrested state, where $0<f_{q} \leqslant 1$. In the latter case, the values $f_{q}$ characterize the arrested glassy state and the $f_{q}$ are called glass-form factors or Debye-Waller factors. In the long-time limit, the equation of motion Eq. (1) reduces to an equation involving only the mode-coupling functional and the glass-form factors [2],

$$
f_{q} /\left(1-f_{q}\right)=\mathcal{F}_{q}[f] .
$$

Frequently studied is the dynamics of a single or tagged particle with the single particle density $\rho_{q}^{s}(t)=\exp \left[i \vec{q}_{s}(t)\right]$. For the correlation function of a tagged particle, $\phi_{q}^{s}(t)=$ $\left\langle\rho_{\vec{q}}^{s *}(t) \rho_{\vec{q}}^{s}\right\rangle$, similar equations as Eqs. (1) have been derived [3, 6],

$$
\tau_{q}^{s} \partial_{t} \phi_{q}^{s}(t)+\phi_{q}^{s}(t)+\int_{0}^{t} m_{q}^{s}\left(t-t^{\prime}\right) \partial_{t^{\prime}} \phi_{q}^{s}\left(t^{\prime}\right) d t^{\prime}=0,
$$

with $\tau_{q}^{s}=1 /\left(D_{0}^{s} q^{2}\right)$. The short-time diffusion coefficient for a single particle, $D_{0}^{s}$, again specifies the Brownian dynamics. The mode-coupling functional for the tagged particle motion,

$$
\mathcal{F}_{q}^{s}\left[f, f^{s}\right]=\int \frac{d^{3} k}{(2 \pi)^{3}} S_{k} \frac{\rho}{q^{4}} c_{k}^{s 2}(\vec{q} \vec{k})^{2} f_{k} f_{|\vec{q}-\vec{k}|}^{s},
$$

is also determined by the static structure of the liquid system where $c_{q}^{s}$ is the single-particle direct correlation function [29].

The dynamics of the tagged particle is coupled to the coherent density correlator $\phi_{q}(t)$ and for that reason $\phi_{q}^{s}(t)$ also displays the bifurcation dynamics that is driven by $\phi_{q}(t)$. The equation for the long-time limits of the tagged particle correlations function, $\phi_{q}^{s}(t \rightarrow \infty)=f_{q}^{s}$, reads

$$
f_{q}^{s} /\left(1-f_{q}^{s}\right)=\mathcal{F}_{q}^{s}\left[f, f^{s}\right] .
$$

In the following, the tagged particle will be assumed as of the same sort as the host fluid. If the host particles are in the liquid state, $f_{q}=0$, a tagged particle cannot be arrested, and in that case Eq. (4) implies $f_{q}^{s}=0$.

The MSD is defined by $\delta r^{2}(t)=\left\langle\left|\vec{r}_{s}(t)-\vec{r}_{s}(0)\right|^{2}\right\rangle$ and describes the average distance a particle has traveled within some time $t$ [29]. It is obtained, e.g., as small wave-number limit of the tagged-particle correlator in Eq. (3), $\phi_{q}^{s}(t)=$ $1-q^{2} \delta r^{2}(t) / 6+O\left(q^{4}\right)[2,6]$,

$$
\delta r^{2}(t)+D_{0}^{s} \int_{0}^{t} m^{(0)}\left(t-t^{\prime}\right) \delta r^{2}\left(t^{\prime}\right) d t^{\prime}=6 D_{0}^{s} t,
$$

$m^{(0)}(t)=\lim _{q \rightarrow 0} m_{q}^{s}(t)=\mathcal{F}_{M S D}\left[\phi(t), \phi^{s}(t)\right] . \quad$ The modecoupling functional for the MSD reads

$$
\mathcal{F}_{M S D}\left[f, f^{s}\right]=\int \frac{d k}{\left(6 \pi^{2}\right)} \rho S_{k}\left(c_{k}^{s}\right)^{2} f_{k} f_{k}^{s} .
$$


A characteristic localization length $r_{s}$ is defined by the second moment for the relaxation of the distribution of $\phi_{a}^{s}(t)$ [2], which can be identified with the functional in Eq. (5b $r_{s}^{2}=1 / \mathcal{F}_{M S D}\left[f, f^{s}\right]$. It is the long-time limit of the MSD. Its value at the critical point, $r_{s}^{c}$, characterizes the arrested structure. The value $6 r_{s}^{c 2}$ represents the plateau for the dynamics of $\delta r^{2}(t)$.

Equations (1) to (5) are solved numerically using algorithms introduced in [30, 31]. Details of the implementation are found in [5, 11]. We use $d$ as unit of length, $d=1$, and choose the unit of time so that $1 / D_{0}=1 / D_{0}^{s}=160$. The structure factors for the SWS and the HCY are calculated in meanspherical approximation [11, 32]. The wave numbers shall be discretized to a grid of $M$ points with a spacing $\Delta q=0.4 / d$. The cutoff in the calculations is ranging from $M=300$ for $\delta>0.04$ up to $M=750$ for $\delta=0.02$.

\section{B. Logarithmic Decay Laws}

The asymptotic solution at higher-order singularities shall be quoted from Ref. [24] where also further details can be found. The asymptotic expansion is performed in small deviations of the correlation function from the critical long-time limit $f_{q}^{c}$ involving the coefficients

$$
A_{q k_{1} \cdots k_{n}}^{(n)}(\mathbf{V})=\frac{1}{n !}\left(1-f_{q}^{c}\right) \frac{\partial^{n} \mathcal{F}_{q}\left[\mathbf{V}, f_{k}^{c}\right]}{\partial f_{k_{1}}^{c} \cdots \partial f_{k_{n}}^{c}}\left(1-f_{k_{1}}^{c}\right) \cdots\left(1-f_{k_{n}}^{c}\right)
$$

which can be split into values at the singularity, $A_{q k_{1} \cdots k_{n}}^{(n) c}$, and remainders, $A_{q k_{1} \cdots k_{n}}^{(n)}(\mathbf{V})=A_{q k_{1} \cdots k_{n}}^{(n) c}+\hat{A}_{q k_{1} \cdots k_{n}}^{(n)}(\mathbf{V})$. The Jacobian matrix of Eq. (2) is singular at the critical points and assumes the form $\left[\delta_{q k}-A_{q k}^{(1) c}\right]$. The non-negative left and right eigenvectors of matrix $A_{q k}^{(1) c}$ shall be denoted by $a_{q}^{*}$ and $a_{q}$ and can be fixed uniquely by requiring $\sum_{q} a_{q}^{*} a_{q}=1$ and $\sum_{q} a_{q}^{*} a_{q}^{2}=1$. The reduced resolvent $R_{q k}$ of $A_{q k}^{(1) c}$ maps vectors orthogonal to $a_{q}^{*}$ to vectors orthogonal to $a_{q}$. The leadingorder result for Eq. (1) is then given by

$$
\phi_{q}(t)=f_{q}^{c}+h_{q}[-B \ln (t / \tau)], B=\sqrt{\left[-6 \varepsilon_{1}(\mathbf{V}) / \pi^{2}\right]},
$$

with the critical amplitudes $h_{q}=\left(1-f_{q}^{c}\right) a_{q}$ and the separation parameter $\varepsilon_{1}(\mathbf{V})=a_{q}^{*} \hat{A}_{q}^{(0)}(\mathbf{V})$, which is restricted to negative values, $\varepsilon_{1}<0$. If $\varepsilon$ indicates the distance of the control parameters $\mathbf{V}$ from the critical point, the leading result is of order $\sqrt{\varepsilon}$ and correct up to $O(\varepsilon)$. The next-to-leading-order approximation is

$$
\begin{aligned}
\phi_{q}(t)=\left(f_{q}^{c}+\hat{f}_{q}\right)+ & h_{q}\left[\left(-B+B_{1}\right) \ln (t / \tau)\right. \\
& +\left(B_{2}+K_{q} B^{2}\right) \ln ^{2}(t / \tau) \\
& \left.+B_{3} \ln ^{3}(t / \tau)+B_{4} \ln ^{4}(t / \tau)\right]
\end{aligned}
$$

what includes the terms of order $\varepsilon$ and neglects terms of order $\varepsilon^{3 / 2}$. It involves corrections to the plateau values,

$$
\hat{f}_{q}=\left(1-f_{q}^{c}\right) R_{q k}\left[\hat{A}_{k}^{(0)}(\mathbf{V})-\varepsilon_{1}(\mathbf{V}) a_{k}^{2}\right]
$$

correction amplitudes,

$$
K_{q}=R_{q k}\left[A_{k k_{1} k_{2}}^{(2) c} a_{k_{1}} a_{k_{2}}-a_{k}^{2}\right] / a_{q}
$$

and the prefactors,

$$
\begin{aligned}
& B_{1}=\left(0.44425 \zeta-0.065381 \mu_{3}\right) \varepsilon_{1}(\mathbf{V})-0.22213 \varepsilon_{2}(\mathbf{V}) \\
& B_{2}=\left(0.91189 \zeta+0.068713 \mu_{3}\right) \varepsilon_{1}(\mathbf{V})-0.15198 \varepsilon_{2}(\mathbf{V}) \\
& B_{3}=-0.13504 \mu_{3} \varepsilon_{1}(\mathbf{V}), \quad B_{4}=-0.046197 \mu_{3} \varepsilon_{1}(\mathbf{V}) .
\end{aligned}
$$

The numbers characterizing the higher-order singularities are

$$
\zeta=\sum_{q} a_{q}^{*}\left[a_{q}^{2} K_{q}+a_{q}^{3} / 2\right]
$$

and

$$
\mu_{3}=2 \zeta-\sum_{q} a_{q}^{*}\left[A_{q k_{1} k_{2} k_{3}}^{(3) c} a_{k_{1}} a_{k_{2}} a_{k_{3}}+2 A_{q k_{1} k_{2}}^{(2) c} a_{k_{1}} a_{k_{2}} K_{k_{2}}\right] .
$$

For the leading correction also an additional separation parameter is introduced,

$$
\begin{array}{r}
\varepsilon_{2}(\mathbf{V})=\sum_{q} a_{q}^{*} \hat{A}_{q k}^{(1)}(\mathbf{V}) a_{k}+2 \varepsilon_{1}(\mathbf{V}) \sum_{q} a_{q}^{*} a_{q}^{2} K_{q} \\
+2 \sum_{q} a_{q}^{*}\left[A_{q k_{1} k_{2}}^{(2) c} a_{k_{1}} \hat{f}_{k_{2}} /\left(1-f_{k_{2}}^{c}\right)-a_{q} \hat{f}_{q} /\left(1-f_{q}^{c}\right)\right] .
\end{array}
$$

The time scale $\tau$ is determined by matching asymptotic approximation and numerical solution of $\phi_{q}(t)$ at the plateau $f_{q}^{c}$ or the rescaled plateau $f_{q}^{c}+\hat{f}_{q}$ for Eq. (7) and Eq. (8), respectively.

\section{Coupled Variables}

Inserting the asymptotic expansion of Eq. (8) into the longtime limit of Eq. (3a), the approximation for the tagged particle dynamics up to order $\varepsilon$ is obtained [33],

$$
\begin{aligned}
\phi_{q}^{s}(t)=\left(f_{q}^{s c}+\hat{f}_{q}^{s}\right)+ & h_{q}^{s}\left[\left(-B+B_{1}\right) \ln (t / \tau)\right. \\
+ & \left(B_{2}+K_{q}^{s} B^{2}\right) \ln ^{2}(t / \tau) \\
& \left.+B_{3} \ln ^{3}(t / \tau)+B_{4} \ln ^{4}(t / \tau)\right]
\end{aligned}
$$

with the critical amplitudes $h_{q}^{s}=\left(1-f_{q}^{s c}\right) a_{q}^{s}$, the correction amplitudes $K_{q}^{s}$ and the plateau corrections $\hat{f}_{q}^{s}$. The latter are derived from the functional $(3 \mathrm{~b})$ and the related coherent quantities by

$$
\sum_{k}\left(\delta_{q k}-A_{q, k}^{s c}\right) a_{k}^{s}=\sum_{k} A_{q k}^{s c} a_{k}
$$




$$
\begin{gathered}
\sum_{k}\left(\delta_{q k}-A_{q, k}^{s c}\right) a_{k}^{s} K_{k}^{s}=-a_{q}^{s 2}+\sum_{k} A_{q k}^{s c} a_{k} K_{k} \\
+\sum_{k, p}\left[A_{q, k p}^{s c} a_{k}^{s} a_{p}^{s}+A_{q k p}^{s c} a_{k} a_{p}+A_{q k, p}^{s c} a_{k} a_{p}^{s}\right] \\
\sum_{k}\left(\delta_{q k}-A_{q, k}^{s c}\right) a_{k}^{s} \hat{f}_{k}^{s}=-\varepsilon_{1}(\mathbf{V}) a_{q}^{s 2}+\sum_{k} A_{q k}^{s c} a_{k} \hat{f}_{k}+\hat{A}_{q}^{s}(\mathbf{V}) .
\end{gathered}
$$

The derivatives with respect to the coherent and tagged particle glass-form factors are denoted before and after the comma, respectively. The coefficients are

$$
\begin{aligned}
& A_{q k_{1} \cdots k_{n}, p_{1} \cdots p_{m}}^{s}(\mathbf{V})=\frac{1}{n !} \frac{1}{m !}\left(1-f_{q}^{s c}\right) \frac{\partial^{n} \partial_{m} \mathcal{F}_{q}^{s}\left[\mathbf{V}, f_{k}^{c}, f_{q}^{s c}\right]}{\partial f_{k_{1}} \cdots \partial f_{k_{n}} \partial f_{p_{1}}^{s} \cdots \partial f_{p_{n}}^{s}} \\
& \quad \times\left(1-f_{k_{1}}^{c}\right) \cdots\left(1-f_{k_{n}}^{c}\right)\left(1-f_{p_{1}}^{s}{ }^{c}\right) \cdots\left(1-f_{p_{n}}^{s}\right)= \\
& =A_{q k_{1} \cdots k_{n}, p_{1} \cdots p_{m}}^{s c}+\hat{A}_{q k_{1} \cdots k_{n}, p_{1} \cdots p_{m}}^{s}(\mathbf{V}) .
\end{aligned}
$$

Similar arguments as above yield the asymptotic expansion for the MSD up to order $\varepsilon$,

$$
\begin{aligned}
\frac{1}{6} \delta r^{2}(t)=r_{s}^{c 2}- & \hat{r}_{s}^{2}-h_{\mathrm{MSD}}\left[\left(-B+B_{1}\right) \ln (t / \tau)\right. \\
& +\left(B_{2}+K_{\mathrm{MSD}} B^{2}\right) \ln ^{2}(t / \tau) \\
& \left.+B_{3} \ln ^{3}(t / \tau)+B_{4} \ln ^{4}(t / \tau)\right]
\end{aligned}
$$

with parameters

$$
h_{\mathrm{MSD}}=r_{s}^{c 4}\left\{\mathcal{F}_{\mathrm{MSD}}^{c}\left[h_{k}, f_{p}^{s c}\right]+\mathcal{F}_{\mathrm{MSD}}^{c}\left[f_{k}^{c}, h_{p}^{s}\right]\right\},
$$

$$
\begin{aligned}
K_{\mathrm{MSD}}=r_{s}^{c 4}\left\{\mathcal{F}_{\mathrm{MSD}}^{c}\left[h_{k}, h_{p}^{s}\right]+\mathcal{F}_{\mathrm{MSD}}^{c}\left[h_{k} K_{k}, f_{p}^{s c}\right]\right. \\
\left.+\mathcal{F}_{\mathrm{MSD}}^{c}\left[f_{k}^{c}, h_{p}^{s} K_{p}^{s}\right]\right\} / h_{\mathrm{MSD}}-h_{\mathrm{MSD}} / r_{s}^{c 2}, \\
\hat{r}_{s}^{2}=r_{s}^{c 4}\left\{\mathcal{F}_{\mathrm{MSD}}^{c}\left[h_{k} \hat{f}_{k}, f_{p}^{s c}\right]+\mathcal{F}_{\mathrm{MSD}}^{c}\left[f_{k}^{c}, h_{p}^{s} \hat{f}_{p}^{s}\right]\right. \\
\left.+\mathcal{F}_{\mathrm{MSD}}^{c}\left[f_{k}^{c}, f_{p}^{s c}\right](\mathbf{V})-\mathcal{F}_{\mathrm{MSD}}^{c}\left[f_{k}^{c}, f_{p}^{s c}\right]\left(\mathbf{V}^{c}\right)\right\} / h_{\mathrm{MSD}} \\
-\varepsilon_{1}(\mathbf{V}) h_{\mathrm{MSD}}^{2} / r_{s}^{c 2} .
\end{aligned}
$$

For the generic liquid-glass transition, the asymptotic expansion was carried out with a different convention as in Eq. (6), however, the quantities $h_{q}, K_{q}, h_{q}^{s}, K_{q}^{s}, h_{\mathrm{MSD}}, K_{\mathrm{MSD}}$, and $\zeta$ are the same in both descriptions [5, 6]. The plateau corrections, $\hat{f}_{q}, \hat{f}_{q}^{s}$, and $\hat{r}_{s}^{2}$ are different for liquid-glass transitions and higher-order singularities. The expansions in Eqs. (8), (15), and (18) share the coefficients $B, B_{1}, B_{2}, B_{3}$, and $B_{4}$. They differ in the plateau and its correction, the critical amplitude and the correction amplitude.

\section{Subdiffusive Power Law in the MSD}

The logarithmic decay laws shall be phrased for the MSD in a slightly different form than in Eq. (18). This is done in order to account for the fact that the MSD is conveniently shown in a double-logarithmic representation which is more sensitive to the detection of power laws. The asymptotic approximation (18) for the MSD can be written as $z=a_{0}+a_{1} y+$ $a_{2} y^{2}+a_{3} y^{3}+a_{4} y^{4}$. Here, $z=\delta r^{2}(t) / 6$ and $y=\ln (t / \tau)$ The constant term represents the square of the corrected localization length, $a_{0}=r_{s}^{c 2}-\hat{r}_{s}^{2}$, the coefficients $a_{1}=h_{\mathrm{MSD}}\left(B-B_{1}\right)$, $a_{2}=-h_{\mathrm{MSD}}\left(B_{2}+K_{\mathrm{MSD}} B^{2}\right)$ as well as $a_{3}=-h_{\mathrm{MSD}} B_{3}$ and $a_{4}-h_{\mathrm{MSD}} B_{4}$ are the separation dependent prefactors for the leading and next-to-leading order terms. This yields the expansion

$\ln z=\ln r_{s}^{c 2}-\hat{r}_{s}^{2} / r_{s}^{c 2}+x^{\prime} y+b_{2} y^{2}+a_{3} / r_{s}^{c 2} y^{3}+a_{4} / r_{s}^{c 2} y^{4}+O\left(\varepsilon^{3 / 2}\right)$,

with

$$
x^{\prime}=a_{1} / r_{s}^{c 2}, \quad b_{2}=\frac{2 r_{s}^{c 2} a_{2}-a_{1}^{2}}{2 r_{s}^{c 4}}
$$

In leading order, one gets a power law for the MSD,

$$
\delta r^{2}(t) / 6=r_{s}^{c 2}(t / \tau)^{x}
$$

with an exponent

$$
x=h_{\mathrm{MSD}} B / r_{s}^{c 2} .
$$

Exponent $x$ varies with the square-root in the separation parameter $\varepsilon_{1}$, cf. Eq. (7). Including the corrections of order $\varepsilon$ rescales the exponent to

$$
x^{\prime}=h_{\mathrm{MSD}}\left(B-B_{1}\right) / r_{s}^{c 2} .
$$

and the next-to-leading order result reads

$$
\begin{aligned}
\delta r^{2}(t) / 6=(t / \tau)^{x^{\prime}} & \left\{r_{s}^{c 2}-\hat{r}_{s}^{2}+b_{2} r_{s}^{c 2} \ln (t / \tau)^{2}\right. \\
& \left.+a_{3} \ln (t / \tau)^{3}+a_{4} \ln (t / \tau)^{4}\right\}
\end{aligned}
$$

\section{CORRELATION FUNCTIONS NEAR AN $A_{4}$-SINGULARITY}

Before we can apply the asymptotic expansion of Eq. 8, we need to specify the values for $\mu_{3}$ and $\zeta$ appearing in the prefactors of Eqs. 11. The $A_{4}$-singularity is characterized by $\mu_{3}=0$. This condition has been used to locate the $A_{4}$ singularity at $\mathbf{V}=\mathbf{V}^{*}$ for the SWS by:

$$
\varphi^{*}=0.52768, \quad \Gamma^{*}=4.4759, \quad \delta^{*}=0.04381 .
$$

The vanishing parameter $\mu_{3}$ implies a considerable simplification in the preceding formulas since $B_{3}=B_{4}=0$ [24]. The deviations of the control parameter values specifying the $A_{4}$ singularity from the ones reported in Ref. [11] originate from refined numerical procedures used here and they do not exceed $6 \%$. The characteristic parameter was $\left|\mu_{3}\right|<5 \cdot 10^{-4}$ at the control-parameter values specified above. The parameter $\zeta$ varies regularly and is $\zeta=0.122$ at the $A_{4}$-singularity. This is smaller than the value in the HSS, $\zeta_{\mathrm{HSS}}=0.269$ [5]. 


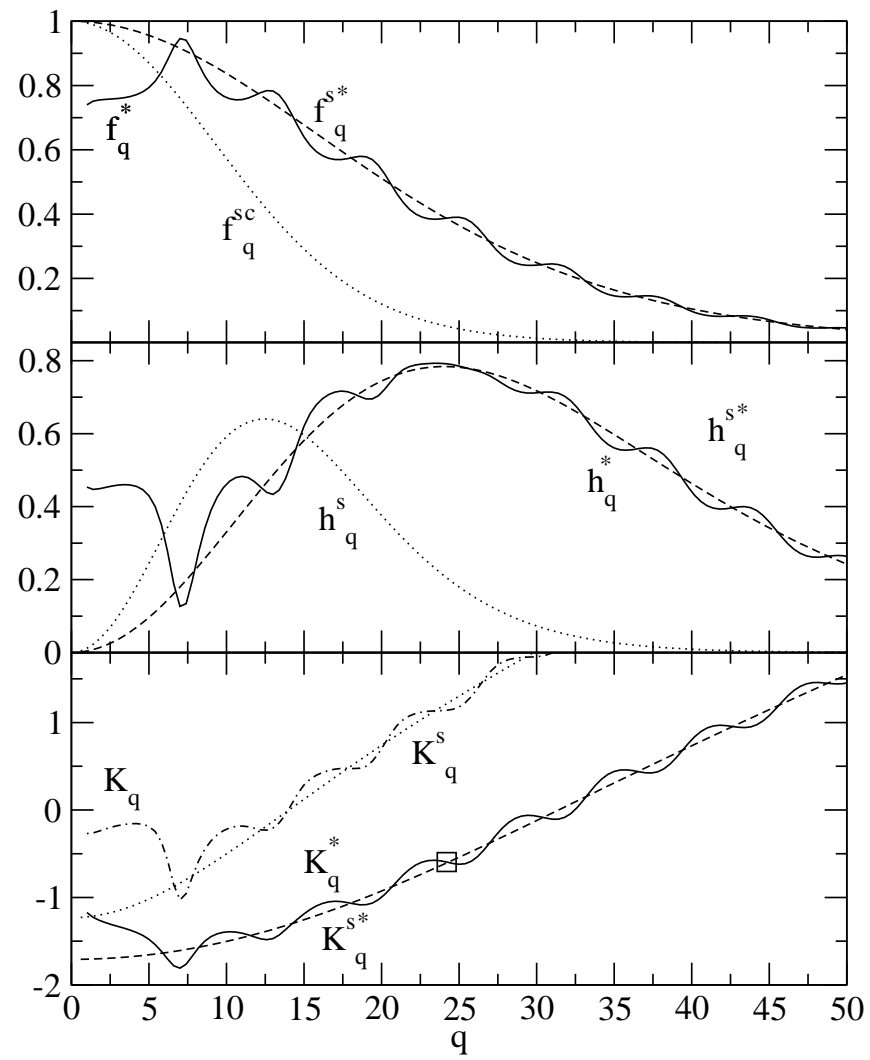

FIG. 1: Wave-vector dependent amplitudes characterizing the $A_{4}$ singularity, Eq. 23, for coherent and tagged particle correlators of the square-well system (SWS). In the upper and middle panel the critical glass-form factors $f_{q}^{*}$, Eq. (2), and the amplitudes $h_{q}^{*}$ are shown as full lines, respectively. The dashed lines represent the values for $f_{q}^{s *}$, Eq. 4], and $h_{q}^{s *}$, Eq. 16a. For the hard-sphere system (HSS), $f_{q}^{s c}$ and $h_{q}^{s}$ are shown dotted. The lower panel shows the correction amplitudes $K_{q}^{*}$, Eq. 10, and $K_{q}^{s *}$, Eq. 16b), as full and dashed lines, respectively. A square at $q=24.2$ indicates the corrections for the path calculated for Fig. 2 and the correlators shown in Fig. 3 The correction amplitudes $K_{q}(-\cdot-)$ and $K_{q}^{s}(\cdots)$ for the HSS are shown for comparison. The unit of length here and in the following figures is the hard-core diameter $d$ of the particles.

The second prerequisite for the asymptotic description according to Eq. (8) are the wave-vector dependent amplitudes $f_{q}^{*}, h_{q}^{*}$ and $K_{q}^{*}$. These are shown for the $A_{4}$-singularity in Fig. 11 together with the related values for the tagged-particle correlator, Eq. [15]. The quantities for the tagged particle motion are close to the ones for the coherent correlator $\phi_{q}(t)$ except for values of $q$ smaller than, say, $q=10$. This difference was observed already for the HSS [6]. Since we will not be concerned with small $q$ in the following, we restrict the discussion to the coherent dynamics and imply that the same is applicable also to the incoherent part with only minor changes. In comparison to the HSS the $f_{q}^{*}, h_{q}^{*}, f_{q}^{s *}$ and $h_{q}^{s *}$ are extended over a broader $q$-range. The maximum in $h_{q}^{s}$ is shifted from $q \approx 13$ to $q \approx 25$ reflecting the smaller localization length in the SWS as noticed before, cf. [11]. We see in the lower panel of Fig. 1 that the distributions of the correction amplitudes $K_{q}$ and $K_{q}^{s}$ share that trend of becoming broader from the HSS to the $A_{4}$-singularity of the SWS. The zero in $K_{q}$ moves from around $q \approx 14$ in the HSS to $q \approx 32$ in the SWS. In addition, the amplitudes are shifted to lower values for small $q$.

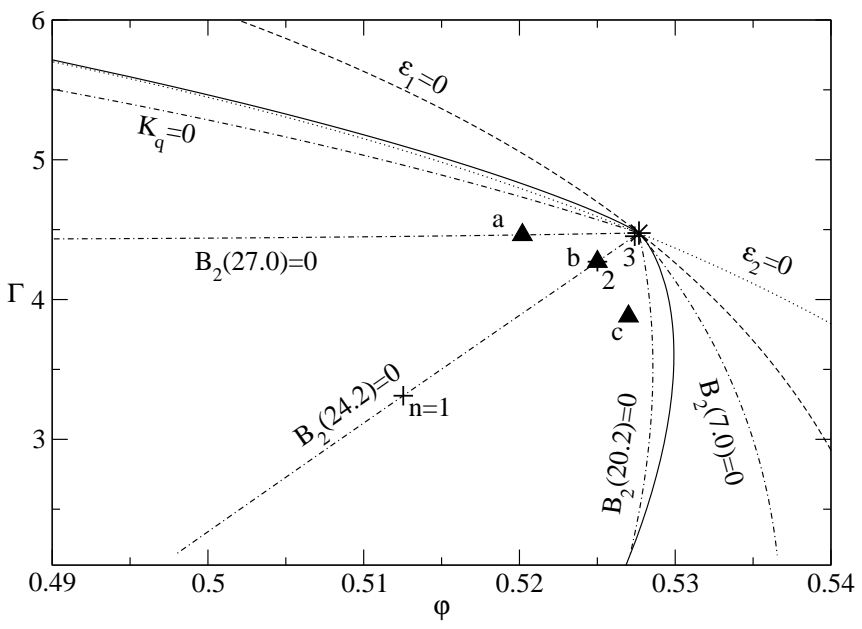

FIG. 2: Curves of vanishing quadratic correction in Eq. 8 at the $A_{4}$ singularity of the SWS, $B_{2}(q)=0$ (dash-dotted), for $q=7.0,20.2$, $24.2,27.0$, and for $K_{q}=0$ as labeled. The full line shows a part of the glass-transition diagram for constant $\delta=\delta^{*}$. The lines of vanishing separation parameters $\varepsilon_{1}(\mathbf{V})$ and $\varepsilon_{2}(\mathbf{V})$ are shown by a broken and a dotted line, respectively. For the wave vector $q=24.2$, a path on the curve $B_{2}(24.2)=0$ is marked ( + ) and labeled by $n$, for which the correlators are shown in Fig. 3 State $n=2$ is analyzed also in Fig. 4 For the points $(\boldsymbol{\Lambda})$ labeled a, b, and c the decay is shown in Figs. 5 and 12

Having specified the characteristic parameters for the $A_{4}$ singularity, the solution at any point in the control-parameter space can be compared to the asymptotic approximation as the control parameters are translated into separation parameters $\varepsilon_{1}$ and $\varepsilon_{2}$. As done for the schematic models in Ref. [24], we start by determining the surfaces where the quadratic corrections in Eq. (8) are zero, $B_{2}(q)=B_{2}+K_{q} B^{2}=0$. On these surfaces in the control-parameter space the logarithmic decay is expected to show up as straight line around the plateau $f_{q}^{*}$, as the cubic and quartic terms in Eq. [8] vanish because of $B_{3}=B_{4}=\mu_{3}=0$ at the $A_{4}$-singularity, cf. Eq. 111. We get a different surface for each wave vector $q$ and show typical examples in Fig. 2 2 for a cut through the glass-transition diagram for $\delta=\delta^{*}$. For $q=7.0$ one gets $K_{q}=-1.81$. The solution of $B_{2}(\mathbf{V})=1.81 B(\mathbf{V})^{2}$ yields the chain line labeled $B_{2}(7.0)=0$ in Fig. 2] and is lying in the arrested region close to the line of liquid-glass transitions. Since the $K_{q}$ depend smoothly on $q$, the evolution of the curve where $B_{2}(q)=0$, can be understood by inspecting the parameters $B$ and $B_{2}$. The square $B^{2}$ is always positive and proportional to $\varepsilon_{1}(\mathbf{V})$, cf. Eq. (7), therefore $K_{q} B^{2}$ is proportional to $K_{q}\left|\varepsilon_{1}(\mathbf{V})\right|$ and shares the sign of $K_{q}$. Inserting $\mu_{3}=0$ and $\zeta=0.1216$ into Eq. (11b) yields $B_{2}(\mathbf{V})=0.111 \varepsilon_{1}(\mathbf{V})-0.152 \varepsilon_{2}(\mathbf{V})$, which has to be positive to comply with $B_{2}(q)=0$. The second separation parameter is negative, $\varepsilon_{2}(\mathbf{V})<0$, below the dotted curve for $\varepsilon_{2}=0$ in Fig. 2 In addition, the value $\left|\varepsilon_{2}(\mathbf{V})\right|$ on the line $B_{2}(7.0)=0$ is larger than $\left|\varepsilon_{1}(\mathbf{V})\right|$ which we can also infer from the fact that the line $\varepsilon_{1}=0$ is closer than the line $\varepsilon_{2}=0$. We now 
chose a point on the line $B_{2}(7.0)=0$, keep the first separation parameter fixed, say $\varepsilon_{1}=\varepsilon_{1}^{\prime}$, and move to higher values for $K_{q}$, e.g., for $q=20.2$ where $K_{q}=-0.966$. $B^{2}$ stays the same and the term $K_{q} B^{2}$ increases. To ensure that $B_{2}(20.2)=0$, the value $B_{2}(\mathbf{V})$ has to decrease. We can achieve that by moving closer to the line $\varepsilon_{2}=0$. For fixed $\varepsilon_{1}^{\prime}$ this implies a shift to lower $\varphi$ and higher $\Gamma$. Consequently the entire line, where $B_{2}(q)=0$, is rotating clockwise around the $A_{4}$-singularity as $K_{q}$ increases. This is seen for the chain line $B_{2}(20.2)=0$ in Fig. 2 Since $K_{q}^{s}$ is monotonic increasing with $q$ and $K_{q}$ has the same trend when neglecting the small oscillations, Fig. 1] the line $B_{2}^{s}(q)=B_{2}(\mathbf{V})+B(\mathbf{V})^{2} K_{q}^{s}=0$ also rotates clockwise with increasing wave-vector $q$.

The variation of the lines $B_{2}(q)=0$ described above depends only on the angle at which the lines $\varepsilon_{1}=0$ and $\varepsilon_{2}=0$ intersect at the $A_{4}$-singularity. This intersection is in a sense generic that it is shared by the close-by $A_{3}$-singularities of the SWS. It applies also to the $A_{4}$-singularities of the other potentials which are similar to the square well. This is so because the functionals determining the separation parameters depend on quantities like the structure factors and the glass-form factors which are similar for different potentials [13]. For a given wave vector $q$, the line $B_{2}(q)=0$ may or may not lie in the liquid regime depending on $K_{q}$. For the SWS at $\delta=\delta^{*}$ we get a range of $-1 \lesssim K_{q} \lesssim 0.4$ corresponding to $20 \lesssim q \lesssim 35$, where a line $B_{2}(q)=0$ is found in the liquid regime. We illustrate this by adding lines for $q=24.2, q=27.0$ and for $K_{q}=0$ to Fig. 22 The vanishing $K_{q}$ is corresponding to $q \approx 32.3$ yielding a line $B_{2}(q)=0$ still in the liquid. For $q \gtrsim 35$, the latter line rotates further around the $A_{4}$-singularity and into the arrested regime beyond the almost horizontal line of liquid-glass transitions.

We select a wave vector $q=24.2$ with $K_{q}=-0.596$ as indicated in Fig. 1 by a square and choose a path on the line $B_{2}(24.2)=0$ marked by the plus symbols in Fig. 2 For $n=$ $1,2,3$, the control parameters are $(\Gamma, \varphi)=(3.312,0.5125)$, $(4.271,0.5250)$, and $(4.453,0.5274)$, respectively. The solutions are shown in Fig. 3 together with the leading approximation, Eq. (7), (dotted) and the next-to-leading approximation, Eq. 8 , (dashed). The time scales $\tau$ are matched at the plateau $f_{q}^{*}$ for the leading approximation and at the renormalized plateaus $f_{q}^{*}+\hat{f}_{q}$ for the first correction. We recognize that for $n=3$, Eq. 8 accounts for more than ten decades in time with a relative accuracy better than 5\%. The leading approximation is acceptable on that level for nine decades. For $n=1$ two and more than one decade are covered, respectively. Five and three orders of magnitude in time are achieved for $n=2$. For $n=1,2,3$, the leading approximation describes at least $30 \%$ of the complete decay and when including the correction, $65 \%$ are covered on the chosen accuracy level. The distance in the control parameter $\Gamma$ from the value at the $A_{4}$-singularity is $25 \%$ for $n=1$ and $4 \%$ for $n=2$, so no fine-tuning was necessary to obtain such large windows for the logarithmic decay. The curve $n=1$ requires about five decades for the complete decay which is well in the reach of today's computer simulation techniques [34].

It was possible to describe part of the critical decay at an $A_{3}$-singularity in a one-component model by the expansion in

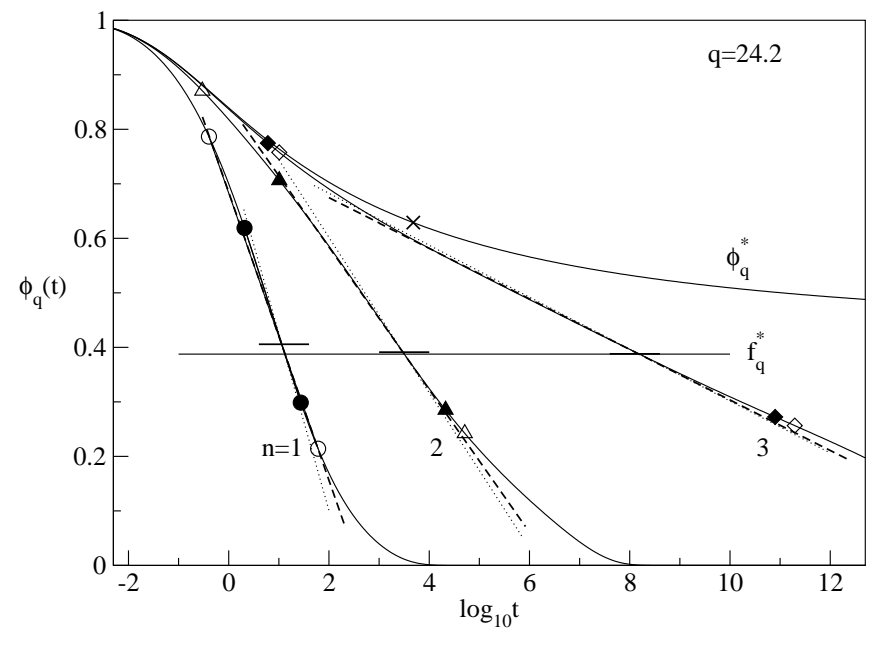

FIG. 3: Logarithmic decay at the $A_{4}$-singularity in the SWS for $q=24.2$ on the path indicated in Fig. 2 The correlation functions are shown as full lines for the states $n=1,2,3$ (see text) and at $\mathbf{V}=\mathbf{V}^{*}$ labeled $\phi_{q}^{*}$. The horizontal line indicates the critical plateau value $f_{q}^{*}$ for $q=24.2$, short lines the renormalized plateaus $f_{q}^{*}+\hat{f}_{q}$. Broken lines show the approximation of Eq. [8, $-\left(B-B_{1}\right) \ln (t / \tau)$, dotted lines the approximation by Eq. (7). Filled and open symbols, respectively, mark the points where the approximations deviate by $5 \%$ from the solution. The cross indicates the time when the solution for $n=3$ and the critical correlator $\phi_{q}^{*}$ differ by $5 \%$. The unit of time here and in the following figures is given by a short-time diffusion coefficient of $D_{0}=1 / 160$.

polynomials in $\ln t$ at a point away from the singularity [24]. We therefore compare the critical decay $\phi_{q}^{*}(t)$ with the decay for $n=3$ and indicate the point at $t \approx 5000$ where both differ by $5 \%$ in Fig. 3 With only the leading correction at our disposal, a $2 \%$-criterion was not fulfilled as for the onecomponent model, where also the next-to-leading correction could be used [24]. The dashed line for $n=3$ does not come closer to the critical decay than $4 \%$. Allowing for 5\%, the interval from $t \approx 20$ to $t \approx 4000$ could be described. However, at the $A_{4}$-singularity the approximation in Eq. (8) always yields a straight $\ln t$-decay as approximation on the chosen path with $B_{2}(q)=0$. This disagrees qualitatively with the observed critical decay.

To identify correctly some decay that is linear in the $\phi_{q}(t)$ versus $\log t$ diagram with the logarithmic decay predicted by the asymptotic laws, Eq. 8 , we check if a different correlator with a different correction amplitude $K_{q}$ is not linear in $\ln t$ at the same point in the control-parameter space. For a twocomponent model a characteristic alternation of concave, linear and convex decay in $\ln t$ was found [24, 33]. Not both correlators could be linear in $\ln t$ at the same time. For the SWS this check is performed at the point $n=2$ from Fig. 2 by variation of the wave vector $q$. For the wave vectors $q=4.2$ and 32.2 the correction amplitudes are $K_{q}=-1.400$ and -0.0413 , respectively. Therefore $B_{2}(4.2)<0$ and $B_{2}(32.2)>0$. We expect $\phi_{q}(t)$ to be concave or convex, accordingly, as is demonstrated by the inset of Fig. 4 The rescaled correlators $\hat{\phi}_{q}(t)$ displayed in the full panel allow for a more detailed analy- 


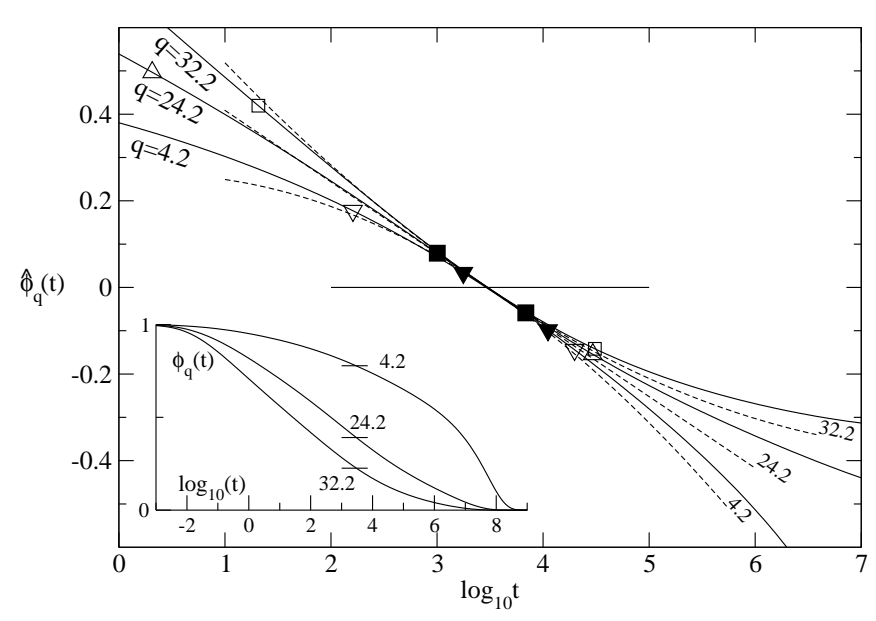

FIG. 4: Logarithmic decay at the $A_{4}$-singularity for varying wave vector $q$. The inset shows the correlation functions $\phi_{q}(t)$ at state $n=2$ from Fig. 2 for wave vectors $q=4.2,24.2$, and 32.2 from top to bottom and the short horizontal lines show the corresponding critical plateau values $f_{q}^{*}$. The full panel shows the same correlation functions rescaled according to $\hat{\phi}_{q}(t)=\left(\phi_{q}(t)-f_{q}^{*}-\hat{f}_{q}\right) / h_{q}^{*}$ as full lines and labeled by the respective wave vectors. Dashed lines show the asymptotic laws, Eq. 8. The deviations of the approximations from the solutions of $5 \%$ are marked by the open symbols. Filled symbols for $q=4.2(\boldsymbol{\nabla})$ and $q=32.2$ ( $\square$ ) show the 5\% deviation from the additional approximation of neglecting quadratic terms in Eq. 8) (see text).

sis. We see that the solutions as well as the approximations clearly exhibit increased curvature for larger $q$. Since the coefficient linear in $\ln t$ is not depending on $q$, cf. Eq. (8), the middle dashed line represents the leading correction to all three correlators when the quadratic terms are neglected. For $q=24.2$ we observe good agreement over almost 5 decades as before, cf. Fig. 3 For $q=4.2$ and 32.2, however, the additional approximation reduces the range of applicability to less than one decade as marked by the filled symbols. Including the quadratic terms from the approximation (8) extends this range by half a decade to later times and to earlier times by one and almost two decades for $q=4.2$ and 32.2, respectively. The time window defined by a $5 \%$ deviation from the approximation 8 is larger by one and two orders of magnitude for $q=24.2$ than for $q=32.2$ and $q=4.2$, respectively, what indicates that $q$-dependent higher-order corrections significantly influence the range of applicability for the leading correction (8).

The time scale $\tau$ in Fig. 4 was matched for $q=24.2$, so the violation of scale universality inherent to an approximation like in Eq. (8) leads to different times $\tau(q)$, where the correlators for different $q$ cross their respective renormalized plateau $f_{q}^{*}+\hat{f}_{q}[24]$. The representation with the rescaled $\hat{\phi}_{q}(t)$ is particularly sensitive to these deviations since the point where the plateau is crossed is required to be zero, $\hat{\phi}_{q}(t / \tau)=0$. In Fig. 4 we see that the line crossing the zero is slightly broader than a single curve. The deviations in $\tau(q)$ are small enough to not exceed the numerical grid for the time axis which around $\tau=2988$ is given by $\Delta t=172$. So we interpolate to get for $q=4.2,24.2$, and 32.2, $\tau(q)=2899,2988$, and 3017, respectively. These differences do not introduce larger errors in the analysis carried out above.

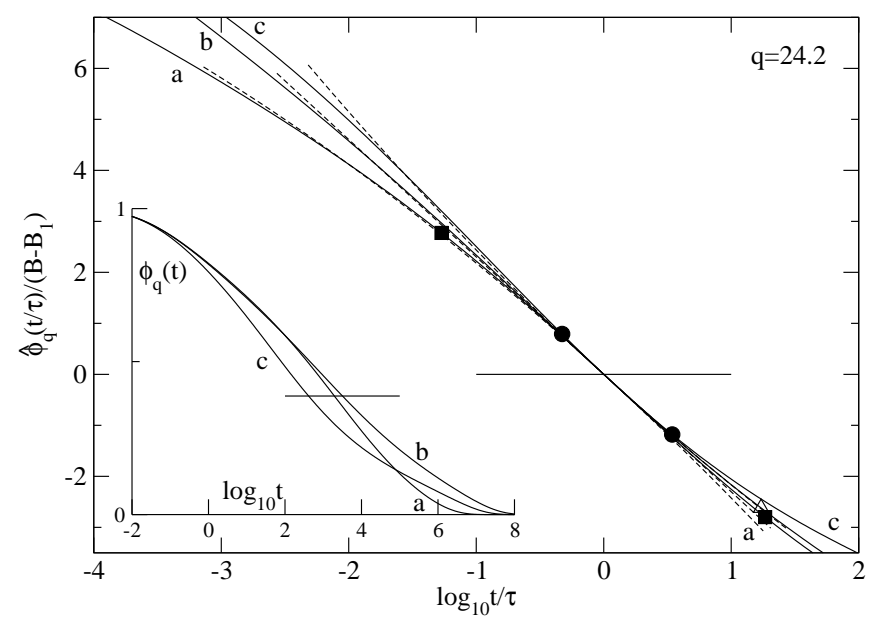

FIG. 5: Logarithmic decay at the $A_{4}$-singularity for the three states marked by triangles in Fig. 2 The inset shows the correlation functions $\phi_{q}(t)$ for $q=24.2$. The plateau value $f_{q}^{*}$ is indicated by the short horizontal line. The full panel shows $\hat{\phi}_{q}(t)=\left(\phi_{q}(t)-f_{q}^{*}-\right.$ $\left.\hat{f}_{q}\right) / h_{q}$ divided by the respective values for $\left(B-B_{1}\right)$ at the three states specified. The dashed curves show the result from Eq. 8]. Filled squares and circles mark the points where curve a and c deviate by $5 \%$ from $-\ln (t / \tau)$, respectively. The deviation for curve $b$ $(\triangle)$ for short times is at $t / \tau \approx 10^{-4}$ and not included in the figure.

In order to change from convex to concave behavior we can also change the control parameters. For states above the line $B_{2}(24.2)=0$, we expect concave behavior, $B_{2}(q)<0$, for states below, convex decay, $B_{2}(24.2)>0$. For a demonstration of this result, the rescaled correlators $\hat{\phi}_{q}(t / \tau)$ at the states labeled a, b, c in Fig. 2 are divided by the prefactor $\left(B-B_{1}\right)$ of Eq. (8). This way the part of the decay that is linear in $\ln t$ shows up as straight line with slope $-\ln 10$ in Fig. 5] The approximations $(8)$ are shown as dashed lines for each state representing $-\ln (t / \tau)+\left[B_{2}(24.2) /\left(B-B_{1}\right)\right] \ln ^{2}(t / \tau)$. For state $\mathrm{b}$ the approximation is identical to $-\ln (t / \tau)$ and the solution follows that line over 5 decades before $5 \%$ deviation is reached. The states a and $c$ are chosen to have the same value for $B-B_{1} \approx 0.015$ and $B_{2}(24.2)=\mp 0.0020$, respectively. The solutions at state a and $\mathrm{c}$ follow the $-\ln t$-law closely within a 5\% margin for two decades or one decade, respectively, which is significantly less than found for state $b$. We can infer from Fig. 2 that at state a the quadratic corrections would vanish again if we went from $q=24.2$ to the higher wave vector $q=27.0$. A scenario similar to the one shown in Fig. 3] can be found.

The procedure outlined in Figs. 2, 3, 4 and 5 can be summarized as follows. From the higher-order singularities there emanate surfaces in the control-parameter space for a specific wave vector $\bar{q}$ where the quadratic term in Eq. 8) is zero, cf. Fig. 2 and the decay is linear in $\ln t$. Moving closer to the singularity on that surface, the window in time where the logarithmic decay is a valid approximation increases, cf. Fig. 3 
On a fixed point on that surface the decay is concave for $q<\bar{q}$ and convex for $q>\bar{q}$, cf. Fig. [4 For fixed $\bar{q}$, the change from concave to convex is achieved by crossing the mentioned surface from above in the sense exemplified in Fig. 5

The coupled quantities share the leading asymptotic behavior of the density correlators. As a consequence of the factorization theorem of MCT, only the glass-form factors and the critical amplitudes $h_{q}$ are different for the coupled quantities [35]. The leading corrections imply a violation of a generalized factorization theorem. These are proportional to the correction amplitude $K_{q}$. Since for large wave vectors, say $q>10$, the quantities $f_{q}^{s}, h_{q}^{s}$, and $K_{q}^{s}$ are close to the ones for the coherent correlator, the approximation for the tagged particle correlation functions $\phi_{q}^{s}(t)$ for these large $q$ is the same as for $\phi_{q}(t)$. So the discussion for $\phi_{q}^{s}(t)$ is already exhausted by Fig. 11 Not much could be gained from repeating the discussion of the previous section for $\phi_{q}^{s}(t)$.

\section{MEAN SQUARED DISPLACEMENT NEAR AN $A_{4}$-SINGULARITY}

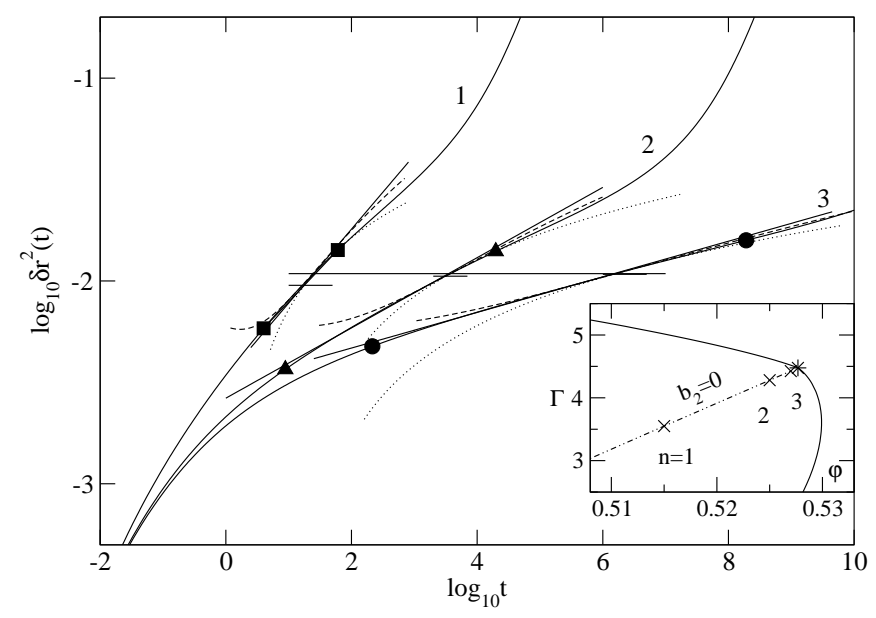

FIG. 6: Subdiffusive power law in the mean-squared displacement (MSD). The solutions for states 1,2, and 3 in the inset are shown as full lines in the full panel together with the leading (dotted) and nextto-leading (dashed) approximation by Eq. 18. The long horizontal line represents $6 r_{s}^{* 2}=0.01086$, the short horizontal lines the corrections to the plateau, $6\left(r_{s}^{* 2}-\hat{r}_{s}^{2}\right)$, cf. Eq. 19c. The straight full lines show the power law $(t / \tau)^{x}$, Eq. 21a, with exponents $x=0.365$, 0.173 and 0.0878 for states $n=1,2,3$. The filled symbols show the points where the solutions deviate by $5 \%$ from the leading-order power laws. The inset shows part of the glass-transition diagram for $\delta=\delta^{*}$ and a chain line where $b_{2}=0$, cf. Eq. 20b, (see text).

According to Eq. 20a,$\delta r^{2}(t)$ is expected to exhibit powerlaw behavior around the plateau $6 r_{s}^{c^{2}}$ provided the term $b_{2}$ vanishes. The power-law exponent $x$ is determined explicitly in Eq. (21b) by the localization length and the critical amplitude, which are

$$
r_{s}^{*}=0.04255, \quad h_{\mathrm{MSD}}^{*}=0.004051 .
$$

The inset of Fig. 6 shows the line where $b_{2}$ from Eq. (20a) vanishes. This line is almost identical to the one for $B_{2}(24.2)=0$ shown in Fig. 2 for the correlators $\phi_{q}(t)$. The MSD for three states on that line is shown in the full panel. It is described well by the approximation in Eq. 18. For states $n=1,2,3$, one, three and six decades are covered with deviations less than 5\%, so the approximation yields a description of similar accuracy as for the correlation functions in Fig. 3 The leading result from Eq. (7) describes the relaxation proportional to $\ln t$ (dotted) which always has negative curvature in the double-logarithmic representation and does not provide a valid description for $n=1$ and 2 . The reason for the qualitative difference between the solution for the MSD and the leading logarithmic law is that the corrections proportional to $K_{\mathrm{MSD}}=-1.708$ are large, Therefore, $K_{\mathrm{MSD}} B^{2}+B_{2}$ is never close to zero in the liquid regime except very close to the $A_{4}$ singularity. This is seen for $n=3$ in Fig. 6 where $\ln t$ develops a straightened decay around the plateau.

The power law 21 provides a different formulation of a leading order approximation and is shown in Fig. 6 as straight line for $n=1,2,3$. For $n=1$ this describes the MSD for more than a decade as indicated by the squares. For $n=2$ three decades are covered and six decades of power-law behavior are identified for curve $n=3$. So the accuracy is similar to the one provided by the approximation in next-to-leading order by Eq. 18. Both asymptotic descriptions fall on top of each other around the plateau and therefore corroborate that the reformulation (20a) is justified. The interpretation of the behavior of the MSD is then much simpler when considering the power laws instead of the logarithms of time. The decreasing slope of the relaxation when approaching the $A_{4}$-singularity as in Fig. 6 is just the exponent $x$ from Eq. (21b) which decreases as $B$ with the square-root of the separation parameter $\varepsilon_{1}$, cf. Eq. (7). The same parameter $B$ is the prefactor of the leading-order logarithmic decay in Eq. (7). In that sense Fig. 6 is the analog of Fig. 3

The term $b_{2}$ in Eq. (20a) varies regularly in the separation parameters $\varepsilon_{1}$ and $\varepsilon_{2}$, and $b_{2}$ is positive above the line $b_{2}=0$ and negative below. Therefore, similar to the case for the correlators in the linear-log representation, in the doublelogarithmic representation, the behavior of the MSD can be changed from convex to concave when crossing the line of vanishing $b_{2}$. This is demonstrated for three states in Fig. 7 State $\mathrm{b}$ is identical to the state $n=2$ in Fig. 6 and obeys $b_{2}=0$. The power law $(t / \tau)^{x}$ is shown as straight full line. The time scale $\tau$ is matched at the plateau $6 r_{s}^{* 2}$. Moving to state $\mathrm{c}$ below the chain line, $(\Gamma, \varphi)=(3.42,0.525)$, a relaxation is obtained which clearly exhibits negative curvature and is consistent with the calculated value $b_{2}=-0.00735$. The leading-order power law with exponent $x=0.285$ fulfills a $5 \%$-deviation criterion for two decades which accidentally extends to short times as the approximation crosses the solution twice. Reducing the allowed deviation to $4 \%$ would reduce that interval to less than a decade. If we include the term proportional to $b_{2}$ from Eq. $22 \mathrm{~b}$ ) and renormalize the exponent to $x^{\prime}$, Eq. 22a , the approximation agrees with the solution for three decades. It is obvious from a comparison with curve 1 in Fig. 6 that the leading-order power law describes that solution better than it describes the solution at state $\mathrm{c}$ in Fig. [7] for 


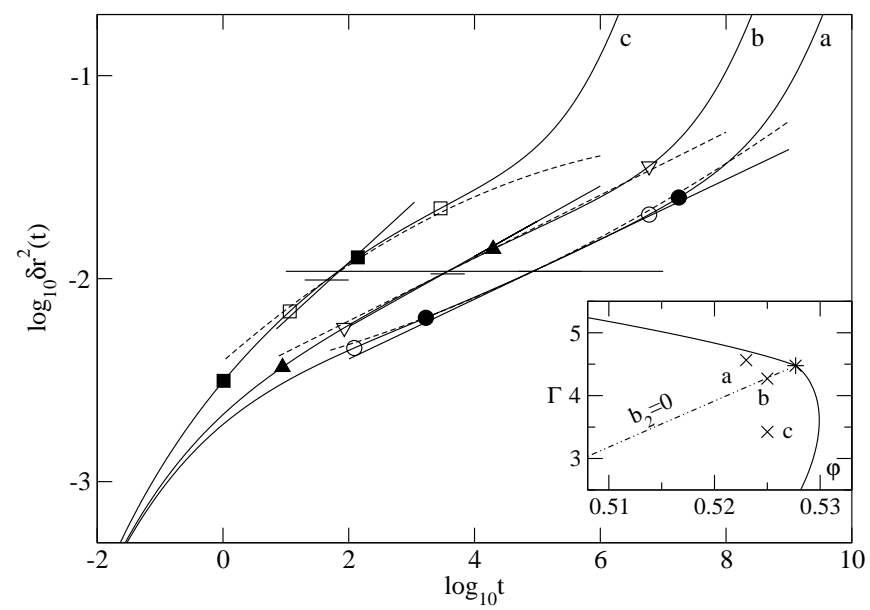

FIG. 7: Concave and convex deviations from the power law, Eq. 21 in the MSD. Solutions for the states $\mathrm{a}, \mathrm{b}$, and $\mathrm{c}$ are shown as full lines, the approximation 21 as straight full lines for exponents $x=0.147$, 0.173 , and 0.285 , respectively. Filled symbols denote the $5 \%$ deviation of the solutions from the leading-order power law. For state b, the dashed line exhibits the corrected power law with $x^{\prime}=0.155, \mathrm{Eq}$. 22a, and the open triangle the 5\% deviations of the solution from it. Dashed lines show the approximation by Eq. 22b for a and c with $b_{2}=0.00363$ and -0.00735 , respectively, and $x^{\prime}=0.143$ and 0.214 . The open symbols mark the 5\% deviations. The inset replots the one from Fig. 6 and shows by the crosses the state points a, b, and c.

comparable values for $\tau$ and the plateau correction $\hat{r}_{s}^{2}$. The deviation to convex behavior is demonstrated by the dashed line at curve $\mathrm{a},(\Gamma, \varphi)=(4.57,0.523)$. Again the range of validity is extended to earlier times but for later times no improvement can be found.

In Fig. 6 the dashed line, which describes the next-toleading order approximation of Eq. (8), deviates from the leading order power law 21a below the plateau where the range of validity for the power law extends to much smaller times than justified by its derivation. We also recognize that the exponent $x$ overestimates the slope of the relaxation in Figs. 6 and 7 In Eq. 21b only the term $B$ from the leading order approximation is present. Taking into account the renormalization of this prefactor to $B-B_{1}$ in Eq. 22a changes the exponent for state $\mathrm{b}$ from $x=0.173$ to $x^{\prime}=0.155$. By comparing the full line for the leading result and the dashed line for the corrected one in Fig. 7 we find that the range of applicability is shifted to later times by one decade and extended by two decades. The corrected power law is valid from $t=10^{2}$ to $t=5 \cdot 10^{6}$ and comparison to Fig. 6 shows that approximation (8) covers a similar range. The accidental extension to shorter times is removed. The approximation now covers the range also a naive power-law fit would yield.

In summary, the correction amplitude $K_{\mathrm{MSD}}$ for the MSD does not vanish within the liquid regime. Therefore a logarithmic relaxation law can be detected only for states very close to the singularity. However, there is a line of vanishing corrections for the logarithm of the MSD. Here a logarithmic relaxation can be observed and this describes a subdiffusive power law of the MSD. We can interpret Fig. [7 as the analog of Fig. 5. Some quadratic correction to a leading-order linear behavior can be set to zero on a surface in control-parameter space. Departing from that surface in opposite directions introduces either positive or negative corrections and the linear behavior is changed to convex or concave.

\section{V. $A_{3}$-SINGULARITIES}

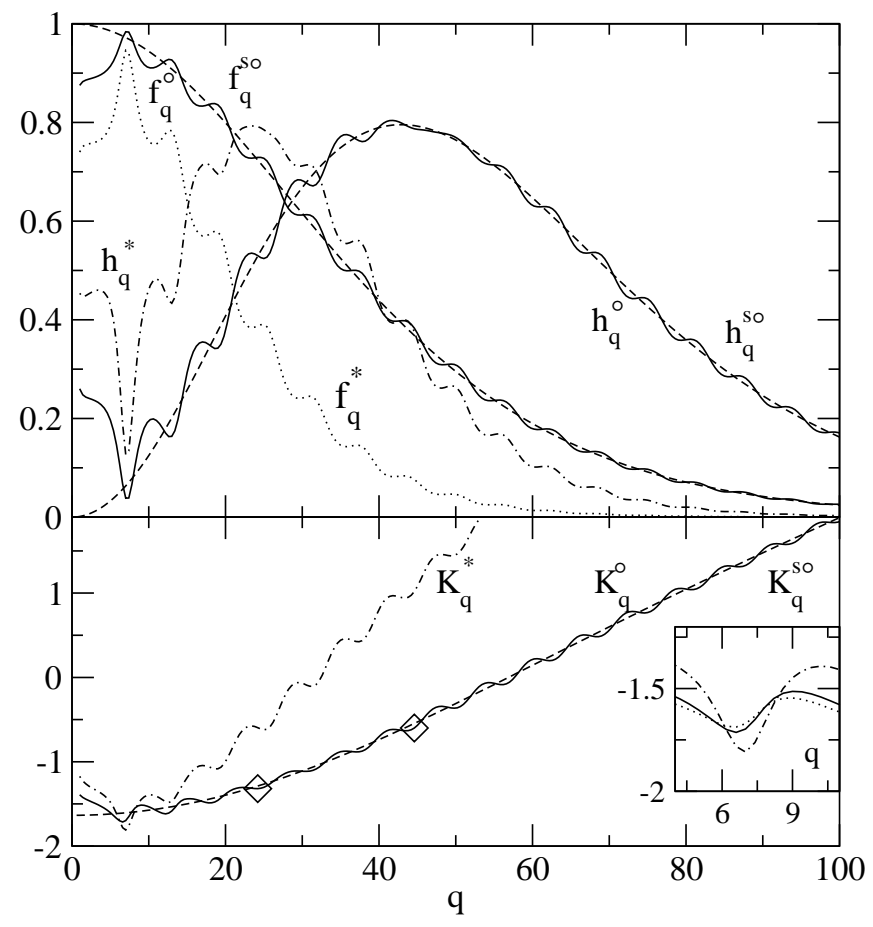

FIG. 8: Glass-form factors $f_{q}^{\circ}$ and $f_{q}^{s \circ}$, amplitudes $h_{q}^{\circ}$ and $h_{q}^{s \circ}$, and correction amplitudes $K_{q}^{\circ}$ and $K_{q}^{s \circ}$ at the $A_{3}$-singularity for $\delta=0.03$. Line styles are the same as in Fig.11 The values at the $A_{4}$-singularity, $f_{q}^{*}$ (dotted), $h_{q}^{*}$ (dash-dotted), and $K_{q}^{*}$ (dash-dotted), are shown for comparison. The values for $q=24.2$ and 45.0 are marked by diamonds. The inset shows $K_{q}$ for $4<q<11$ for $\delta=\delta^{*}$ (chain line), 0.03 (full line) and 0.02 (dotted line).

An $A_{3}$-singularity is not located on a liquid-glass-transition line but is the endpoint of a glass-glass-transition line [2]. The parameter $\mu_{3}$ is no longer vanishing and for $\delta=0.03$ we get $\mu_{3}=0.109$ and $\zeta=0.157$. For this $A_{3}$-singularity the $q$-dependent amplitudes are shown in Fig. 8 No qualitative changes are obvious compared to the results shown in Fig. 1 for the $A_{4}$. The smaller length scale $\delta=0.03$ for the attractive well introduces a smaller localization length, and this implies the broader distributions in wave-vector space. So the trend seen when changing from the HSS to the $A_{4}$-singularity of the SWS is continued when approaching $A_{3}$-singularities at smaller $\delta$. There are only two notable exceptions at smaller $q$. First, the value for $K_{q}$ at the position of the structure factor peak is minimal for the $A_{4},-1.81=K_{q}^{*}<K_{q}^{\circ}=-1.72$. The inset shows this region enlarged for $\delta=\delta^{*}, 0.03$ and 0.02 , demonstrating that $K_{q}$ at the peak is again larger for the $A_{3}$ singularity with smaller well width 0.02 , where $K_{q}=-1.69$. 
Second, the zero-wave-vector limit of $K_{q}^{s}$ is also smallest at the $A_{4}$-singularity. The respective values for $\delta=\delta^{*}, 0.03$ and 0.02 are $-1.71,-1.64$, and -1.62 . Therefore, one experiences the strongest $q$-dependent corrections at the $A_{4}$-singularity.

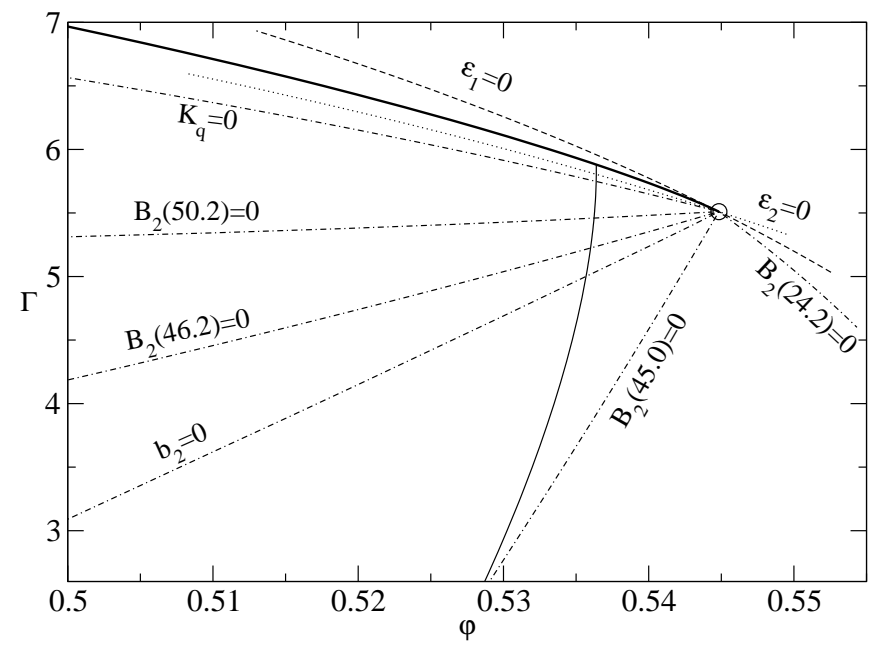

FIG. 9: Curves of vanishing quadratic correction in Eq. 8 for the $A_{3}$-singularity $(\bigcirc)$ at $\delta=0.03$. The $\delta=0.03$ cut through the glasstransition diagram is displayed by full lines. The various lines are shown in the same style as in Fig. 2 and labeled accordingly. The line $b_{2}=0$, cf. Eq. 20b, indicates the analogous line for the MSD, cf. inset of Fig. 6

Figure 9 shows the analog of Fig. 2 for a cut through the glass-transition diagram at $\delta=0.03$. The lines $\varepsilon_{1}=0$ and $\varepsilon_{2}=0$ for the $A_{3}$-singularity are obtained from a smooth transformation of the corresponding lines at the $A_{4}$-singularity, and they appear in similar locations in the diagram. The line $\varepsilon_{2}=0$ is again very close to the almost horizontal line of transitions. Just below, we find again the line where $B_{2}(q)=0$ when $K_{q}=0$. However, this now represents $q \approx 57.5$, cf. Fig. 8 which is a value almost twice as large as for the corresponding line in Fig. 2 For the wave vector $q=24.2$ we find the line, where $B_{2}(24.2)=0$, completely in the glass state. Taking the same value for the correction amplitude as for $q=24.2$ at the $A_{4}, K_{q}^{*} \approx-0.6$, we obtain $q=45.0$, cf. Fig. 8 and the line labeled accordingly in Fig. 9 Since the latter line comes close to the liquid-glass-transition line we take that as a reference and estimate the range of wave-vectors where the quadratic corrections can be put to zero in the liquid regime to $45 \lesssim q \lesssim 70$. The lines where $B_{2}(q)=0$ can be rather sensitive to $q$-variation. This is demonstrated by the curve $B_{2}(46.2)=0$. Although the change in the wave vector is relatively small in comparison to $q=45.0$, the values for $K_{q}$ differ by more than $20 \%$ for fixed $q$ and induce a rotation of the line $B_{2}(q)=0$ by quite a significant angle.

Having in mind the drastic changes in the lines where $B_{2}(q)=0$, it may come with some surprise that the line for the MSD, where $b_{2}=0$, stays rather robust and accessible in the liquid regime as seen in Fig. 9 The variation in $q$ for the amplitudes is reflected in changes of the localization lengths.
For the $A_{3}$-singularity at $\delta=0.03$ we get

$$
r_{s}^{\circ}=0.0243, \quad h_{\mathrm{MSD}}^{\circ}=0.00136
$$

From Eq. 24) one gets $r_{s}^{*} / r_{s}^{\circ}=1.75$ and the square of the latter ratio, $r_{s}^{* 2} / r_{s}^{\circ 2} \approx 3$, is the same as $h_{\mathrm{MSD}}^{*} / h_{\mathrm{MSD}}^{\circ}$. Since only the fraction $h_{\mathrm{MSD}} / r_{s}^{2}$ could introduce larger modifications in Eq. (20a), the changes in $b_{2}$ cancel approximately and the line specified by $b_{2}=0$ experiences only minor deformations when $\delta$ is varied. The wave vector for which the lines $B_{2}(q)=0$ and $b_{2}=0$ are closest to each other, is $q=45.8$ at the $A_{3}$-singularity for $\delta=0.03$.

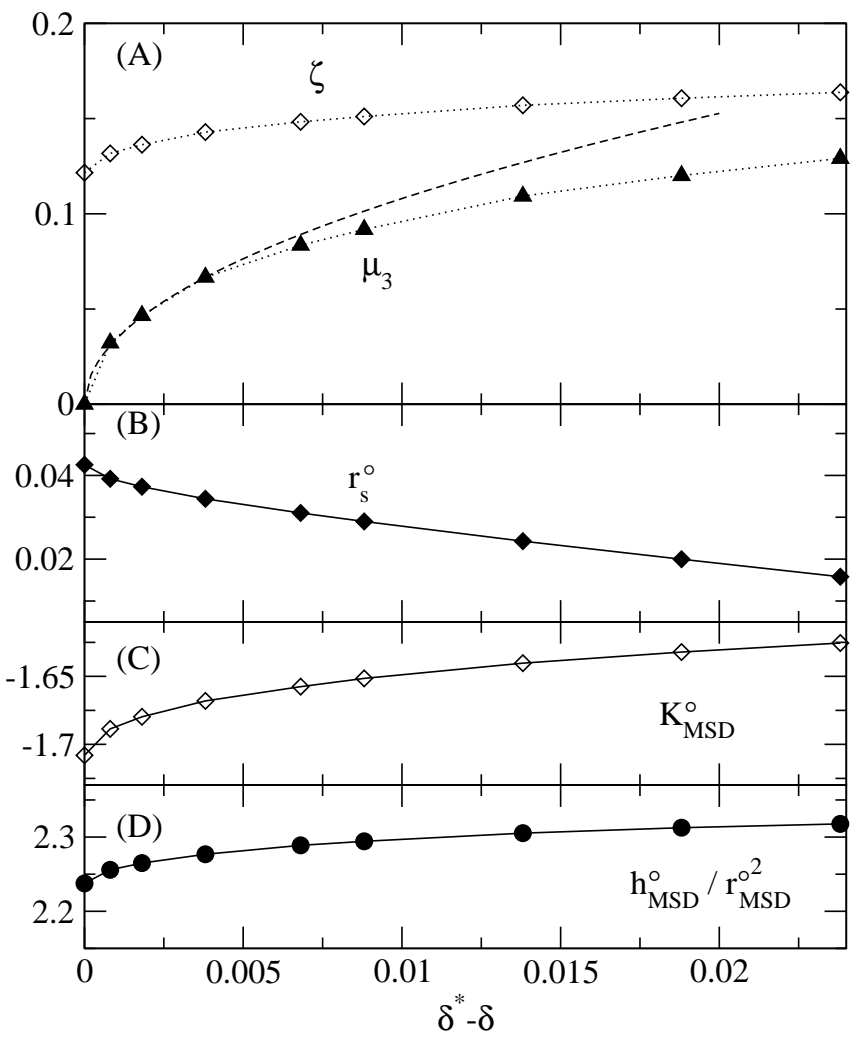

FIG. 10: Parameters for the asymptotic description at the $A_{3}$ singularities of the SWS for varying $\delta$. Panel A displays $\mu_{3}(\boldsymbol{\Delta})$, Eq. 13, and $\zeta(\diamond)$, Eq. 12). The dashed curve shows the asymptotic $\sqrt{\delta^{*}-\delta}$-law for the $\mu_{3}$. The localization length $r_{s}^{\circ}$ is shown in panel B. The correction amplitudes $K_{\mathrm{MSD}}^{\circ}$ and the ratios $h_{\mathrm{MSD}}^{\circ} / r_{\mathrm{MSD}}^{\circ 2}$ are shown in panels $\mathrm{C}$ and $\mathrm{D}$.

To corroborate the finding for the MSD from the preceding paragraph, the parameters for the asymptotic description of the MSD at the $A_{3}$-singularities are shown in Fig. 10 The $\mu_{3}$ vanish when we approach the $A_{4}$-singularity. The decrease close to $\delta^{*}$ is described asymptotically by a square-root variation, $\mu_{3} \propto \sqrt{\delta^{*}-\delta}$, shown by the dashed line [33]. The smallness of $\mu_{3}$ indicates that all the $A_{3}$-singularities are already influenced by the proximity of the close-by $A_{4}$-singularity. One can take advantage of this finding and conclude that the terms proportional to $\mu_{3}$ in Eq. (8) are small. Moreover, one may neglect $B_{3}$ and $B_{4}$ in Eq. $11 \mathrm{c}$ entirely without introducing large additional errors. The leading correction to the logarithmic 
decay laws is then only quadratic also for the $A_{3}$-singularities. Parameter $\zeta$ varies regularly around a finite value at $\delta^{*}$ but shares the variation of $\mu_{3}$ at $\delta^{*}$ due to Eq. 13. Panel B shows the decrease of the localization length at the $A_{3}$-singularity when $\delta$ is reduced. A change of $40 \%$ in $r_{s}^{\circ}$ from $\delta=\delta^{*}$ to $\delta=0.03$, cf. Eqs. 24 and 25, is reflected in the broadening of the distributions in $q$ seen in Figs. 1 and 8 This broadening is responsible for the large variation in $q$ when comparing Fig. 2] with Fig. 9 It was noted in the discussion of the inset of Fig. 8 that $K_{q}$ introduces the strongest corrections for the correlation functions at the $A_{4}$-singularity. This is also true for the MSD as seen in panel $\mathrm{C}$ for $K_{\mathrm{MSD}}$ which is largest in absolute value at the $A_{4}$-singularity. The variation in $K_{\mathrm{MSD}}$ with $\delta$ is however small and does not introduce significant changes to $a_{2}$ in Eq. (20b). The amplitude $h_{\mathrm{MSD}}$ is the remaining parameter entering Eq. 20b that could alter the location of the line $b_{2}=0$ in the glass-transition diagram. We noted above that only the ratio $h_{\mathrm{MSD}} / r_{s}^{c 2}$ needs to be considered which is shown in panel D. From there one infers that the ratio varies only by less than $5 \%$. We can conclude that the line of power law variation for the MSD stays in the liquid regime even when $\delta$ is changed significantly.

\section{HARD CORE YUKAWA SYSTEM}

The $A_{l}$-singularities occurring in MCT are topologically stable, smooth changes in the control parameters do not challenge their existence. Therefore, the results for the SWS can be applied also to other potentials with a short-ranged attraction. Nevertheless, the deformation of the potential might introduce changes large enough to be relevant for the detection of the higher-order singularities. Among several potentials the hard core Yukawa system (HCY) was found to differ by up to $20 \%$ in certain properties at the $A_{4}$-singularity from the SWS [13]. Since other potentials differ less we use that system as a second example for an $A_{4}$-singularity.

Figure 11 shows the analog of Fig. 2 for the HCY. For a comparison, the $A_{4}$-singularity in the SWS was mapped on top of the $A_{4}$-singularity in the HCY by scaling in $\Gamma$ with a factor of 2.98 and by a shift in $\varphi$ of 0.0065 . The same transformation was applied to the lines where $B_{2}(q)=0$ in the SWS. Fig. 11 displays the $B_{2}(q)=0$ lines for the HCY that come closest to the ones shown in Fig. 2] after the mapping. The correction amplitude $K_{q}$ for the $\mathrm{HCY}$ vanishes at $q \approx 34$, and the range in wave vector for which $B_{2}(q)=0$ is lying in the liquid regime is shifted to higher wave vectors, $21 \lesssim q \lesssim 36$ or $-0.9 \lesssim K_{q} \lesssim$ 0.2 , in comparison to the SWS. For $q=27.0$ we get the line $B_{2}(q)=0$ for the HCY that is closest to the line $b_{2}=0$ for the MSD as compared to $B_{2}(24.2)=0$ in the SWS.

\section{CONCLUSION}

Logarithmic decay or, equivalently, $1 / f$ noise in the fluctuation spectra, can arise in a number of situations and is explained by various approaches [36]. In the log-linear representation appropriate for the correlation functions, this decay

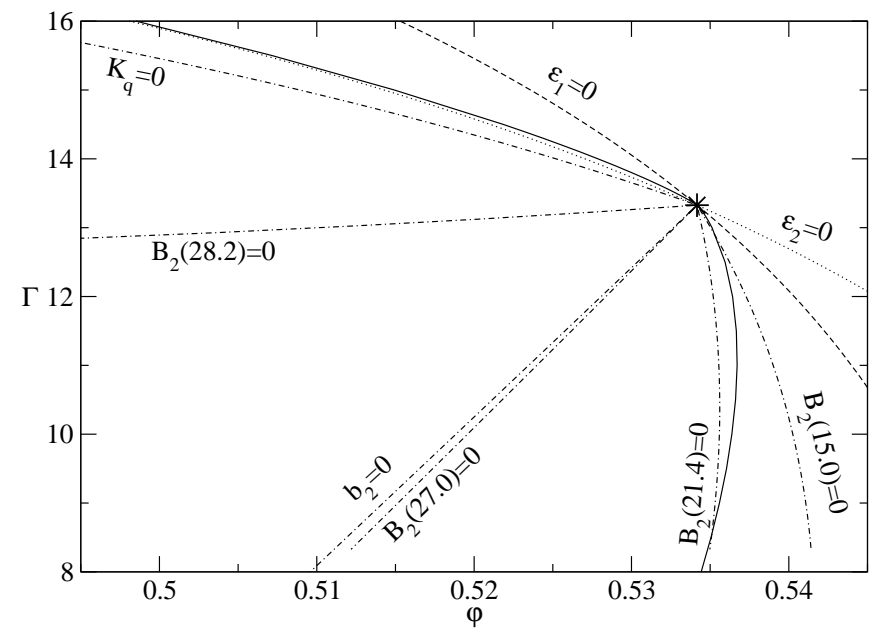

FIG. 11: Cut through the parameter space for the hard-core Yukawa system for $\delta=\delta^{*}$. Lines styles are the same as in Fig. 2] The wave vectors $q=15.0,21.4,27.0$, and 28.2 are approximately equivalent to $q=7.0,20.2,24.2$, and 27.0 in Fig. 2 respectively, after rescaling $\Gamma$ by a factor of 2.98 (see text).

exhibits a straight line. To discriminate the logarithmic decay laws originating from higher-order glass-transition singularities within MCT [24] from other possible scenarios one needs criteria to distinguish one from the other. The theory makes specific predictions where in the control-parameter space the logarithmic decay is expected and how the corrections introduce deviations from that behavior. In this paper, the scenarios are discussed in quantitative detail for an example relevant for studies of colloidal dynamics, the square-well system (SWS). To proceed, specific cuts through the three-dimensional parameter space are considered. Here, lines are identified where the corrections quadratic in the logarithm of time vanish for a chosen wave vector $q$, cf. Fig. 2] These lines emanate from the higher-order singularity and rotate clockwise around the higher-order singularity with increasing $q$. The correlation functions for states on these lines exhibit decays that are linear in the logarithm of time for several orders of magnitude in time, cf. Fig. 3 In leading order, the slope of the decay is given by the square-root of the distance from the higher-order singularity, Eq. (7). The mean-squared displacement MSD displays a power law, Eq. 21a, that is valid on a similar line in the control-parameter space, cf. Fig. 6 The exponent $x$ of this subdiffusive behavior is also decreasing with the squareroot of the distance. Both the logarithmic decay and the power law are accessible in the liquid regime. The logarithmic decay is predicted for wave vectors $q$, which are equivalent to values of about three to four times the first peak of the static structure factor.

In a semi-logarithmic representation for the correlation functions and a double-logarithmic plot of the MSD, characteristic convex and concave relaxation patterns are found when states are chosen that are off the specified lines, cf. Figs. 5 and 7 Due to the variation of the correction amplitude $K_{q}$ in Fig. 11 a similar variation from convex to concave behavior is introduced by changes in the wave vector at a fixed 
point in control-parameter space, cf. Fig. 4 These deviations from logarithmic behavior provide a test for the clear identification of dynamical scenarios that are consistent with Eq. 8) and hence originate from higher-order singularities.

When the localization at the higher-order singularity is changed by either deforming the shape of the potential or by moving to $A_{3}$-singularities at smaller ranges of the attraction, the logarithmic decay of the correlation functions is shifted to higher wave vectors. Whereas the difference between the SWS and the hard-core Yukawa system at the $A_{4}$-singularity is modest, cf. Fig. 2 and Fig.11 the lines of vanishing quadratic correction change drastically at the $A_{3}$-singularity, cf. Fig. 9 In contrast, the line where the subdiffusive power law for the MSD is valid, is robust against changes of the well width and the potential shape, cf. Figs. 6 9 and 11

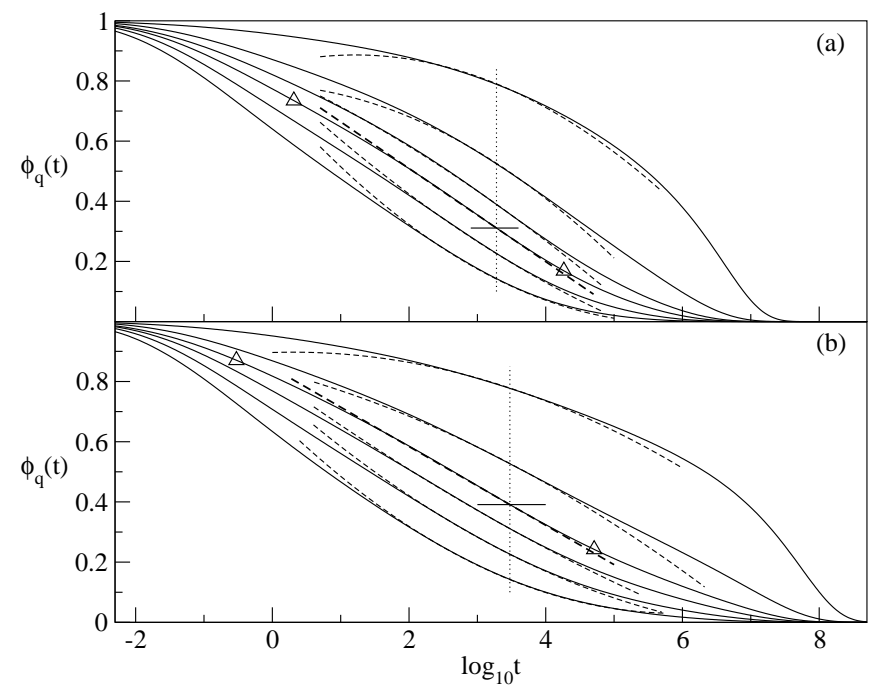

FIG. 12: Correlators for states a (panel a) and b (panel b) from Fig. 2 for wave vectors $q=4.2,20.2,24.2,27.0,32.2$, and 36.2 from top to bottom. Full lines show the solutions of the MCT equations for the SWS, dashed lines the approximation by Eq. 8. Triangles mark the 5\% deviation of the correlator from the approximation for $q=27.0$ and 24.2 , respectively. The dotted vertical lines indicate the time scales $\tau$, the short horizontal lines the corrected plateau value $f_{q}+\hat{f}_{q}$ for $q=27.0$ and 24.2 , respectively.

For comparing the solutions of the equations of motion, Eqs. (1), (3), and (5), with the asymptotic expansions, Eqs. (8), (15), and (18), all parameters are calculated explicitly except the time scale $\tau$ which is matched at the plateau. In an experiment or a computer simulation only the correlators are available directly. We show these in Fig. 12 for two states specified in Fig. 2 for different wave vectors. Since state b is closer to the $A_{4}$-singularity, the range of validity for the asymptotic approximation is larger than for state a. Especially the extension of the linear-log decay at some specific wave vector increases when moving closer to the singularity. As noted in connection with Fig. 4 the range of validity for the approximation by Eq. (8) may vary with $q$. Partly for that reason a larger absolute curvature is attributed to the correlators by the approximation than a fit would do. A free fit could identify logarithmic behavior at state $\mathrm{b}$ for $q=20.2$ from $t \approx 5$ to $t \approx 5 \cdot 10^{7}$ with a deviation of at most $5 \%$. In addition, fitting the correlator for $q=24.2$ also for $t \geqslant 10^{5}$ would yield positive curvature. Therefore, a free fit in that region of the control-parameter space tends to find the logarithmic decay at a somewhat lower wave vector than predicted by Eq. (8). However, with a choice of the time scale $\tau$ that is reasonably close to the theoretical value, the concave and convex decay patterns can still be identified unambiguously in the correlators without invoking additional assumptions.

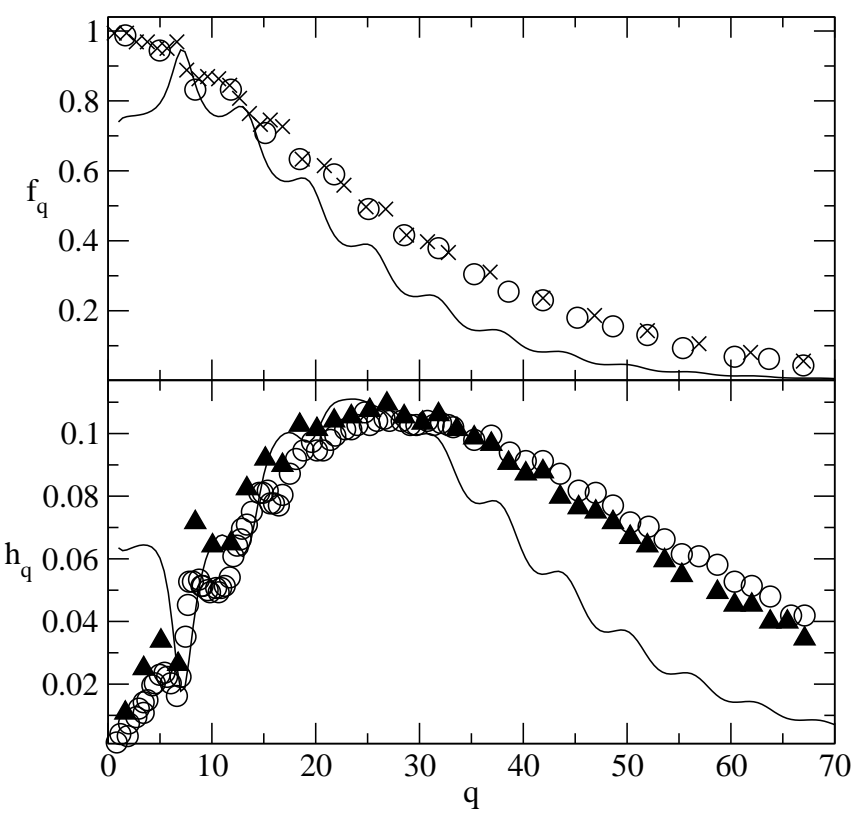

FIG. 13: Comparison of $f_{q}$ and $h_{q}$ from the fit to the simulation of two different states [37] with the values $f_{q}^{*}$ and $h_{q}^{*}$ for the SWS from Fig.1 For the comparison in the lower panel the theoretical values are multiplied by 0.14 .

A recent molecular dynamics study of a binary mixture of square-well particles identifies a power law with $x=0.28$ for the MSD over four decades and a related logarithmic decay of the correlation function at a wave vector $q=16.8$ [37]. A scenario similar to Fig. 12 was found for the correlation functions: Upon increasing $q$, a change from concave to convex decay is observed. For a second state, faster decay with larger prefactors for the logarithmic decay is reported together with a larger exponent, $x=0.44$, for the power law in the MSD. This finding is consistent with the assumption that this second state is further from the supposed higher-order singularity than the first state. Different from Fig. 12 in the simulation $\delta$ was changed to vary the distance while $\varphi$ and $\Gamma$ were kept fixed. The logarithmic decay was shifted to a higher wave vector for smaller $\delta$ [37]. This is consistent with the expectation that can be inferred from Figs. 2 and 9 by observing, e.g., the rotation of the line $B_{2}(24.2)=0$. The analysis of the simulation data allowed for a fit of the values for $f_{q}^{*}$ and $h_{q}^{*}[37]$. These are shown in Fig. 13 together with the theoretical predictions for the SWS. The fitted parameters for both states almost fall on top of each other for $f_{q}^{*}$. The amplitude $h_{q}^{*}$ is deduced from the simulation data only up to some overall factor. It can be 
matched reasonably by a multiplication of the theoretical prediction for $h_{q}^{*}$. The extension in $q$ for the values obtained from MCT for the SWS are narrower, the width at half maximum for $f_{q}$ differs by $15 \%$. A similar difference was observed for a binary mixture of hard spheres and agreement between theory and simulation could be improved by using the structure factor from the simulation as input to the MCT calculations [38]. For the amplitude $h_{q}^{*}$ the locations of the maxima disagree by $15 \%$ and the width is different by $25 \%$. The deviations for $q<7$ in both $f_{q}$ and $h_{q}$ can be attributed to the effects of mixing [38].

In summary, scenarios for logarithmic decay near higherorder glass-transition singularities are presented in this work.
Some essential predictions are supported by the results of computer simulations. This should motivate further investigations in colloidal systems with short-ranged attraction. In particular the power-law behavior for the MSD including the deviations might be accessible to experiments.

\section{Acknowledgments}

I thank W. Götze for valuable discussion. This work was supported by the Deutsche Forschungsgemeinschaft Grant Go154/13-1.
[1] J.-P. Boon and S. Yip, Molecular Hydrodynamics (McGrawHill, New York, 1980).

[2] W. Götze, in Liquids, Freezing and Glass Transition, edited by J. P. Hansen, D. Levesque, and J. Zinn-Justin (North Holland, Amsterdam, 1991), vol. Session LI (1989) of Les Houches Summer Schools of Theoretical Physics, pp. 287-503.

[3] U. Bengtzelius, W. Götze, and A. Sjölander, J. Phys. C 17, 5915 (1984).

[4] V. I. Arnol'd, Catastrophe Theory (Springer, Berlin, 1992), 3rd ed.

[5] T. Franosch, M. Fuchs, W. Götze, M. R. Mayr, and A. P. Singh, Phys. Rev. E 55, 7153 (1997).

[6] M. Fuchs, W. Götze, and M. R. Mayr, Phys. Rev. E 58, 3384 (1998).

[7] P. N. Pusey and W. van Megen, Nature 320, 340 (1986).

[8] W. van Megen, Transp. Theory Stat. Phys. 24, 1017 (1995).

[9] L. Fabbian, W. Götze, F. Sciortino, P. Tartaglia, and F. Thiery, Phys. Rev. E 59, R1347 (1999); 60, 2430(E) (1999).

[10] J. Bergenholtz and M. Fuchs, Phys. Rev. E 59, 5706 (1999).

[11] K. Dawson, G. Foffi, M. Fuchs, W. Götze, F. Sciortino, M. Sperl, P. Tartaglia, T. Voigtmann, and E. Zaccarelli, Phys. Rev. E 63, 011401 (2001).

[12] W. C. K. Poon, J. Phys.: Condens. Matter 14, R859 (2002).

[13] W. Götze and M. Sperl, J. Phys.: Condens. Matter 15, S869 (2003).

[14] T. Eckert and E. Bartsch, Phys. Rev. Lett. 89, 125701 (2002).

[15] K. N. Pham, A. M. Puertas, J. Bergenholtz, S. U. Egelhaaf, A. Moussaïd, P. N. Pusey, A. B. Schofield, M. E. Cates, M. Fuchs, and W. C. K. Poon, Science 296, 104 (2002).

[16] G. Foffi, K. A. Dawson, S. V. Buldyrev, F. Sciortino, E. Zaccarelli, and P. Tartaglia, Phys. Rev. E 65, 050802 (2002).

[17] E. Zaccarelli, G. Foffi, K. A. Dawson, S. V. Buldyrev, F. Sciortino, and P. Tartaglia, Phys. Rev. E 66, 041402 (2002).

[18] A. M. Puertas, M. Fuchs, and M. E. Cates, Phys. Rev. E 67,
031406 (2003).

[19] A. M. Puertas, M. Fuchs, and M. E. Cates, Phys. Rev. Lett. 88, 098301 (2002).

[20] F. Mallamace, P. Gambadauro, N. Micali, P. Tartaglia, C. Liao, and S.-H. Chen, Phys. Rev. Lett. 84, 5431 (2000).

[21] W. K. Kegel and A. van Blaaderen, Science 287, 290 (2000).

[22] E. R. Weeks, J. C. Crocker, A. C. Levitt, A. Schofield, and D. A. Weitz, Science 287, 627 (2000).

[23] E. R. Weeks and D. A. Weitz, Phys. Rev. Lett. 89, 095704 (2002).

[24] W. Götze and M. Sperl, Phys. Rev. E 66, 011405 (2002).

[25] G. Szamel and H. Löwen, Phys. Rev. A 44, 8215 (1991).

[26] M. Fuchs, Ph.D. thesis, TU München (1993).

[27] W. Götze and M. Lücke, Phys. Rev. B 13, 3825 (1976).

[28] L. Sjögren, Phys. Rev. A 22, 2883 (1980).

[29] J.-P. Hansen and I. R. McDonald, Theory of Simple Liquids (Academic Press, London, 1986), 2nd ed.

[30] M. Fuchs, W. Götze, I. Hofacker, and A. Latz, J. Phys.: Condens. Matter 3, 5047 (1991).

[31] W. Götze, J. Stat. Phys. 83, 1183 (1996).

[32] P. T. Cummings and E. R. Smith, Chem. Phys. 42, 241 (1979).

[33] M. Sperl, Ph.D. thesis, TU München (2003).

[34] W. Kob, in Slow Relaxations and Nonequilibrium Dynamics in Condensed Matter, edited by J.-L. Barrat, M. Feigelman, and J. Kurchan (Springer, Berlin, 2003), vol. Session LXXVII (2002) of Les Houches Summer Schools of Theoretical Physics.

[35] W. Götze, Z. Phys. B 60, 195 (1985).

[36] M. B. Weissman, Rev. Mod. Phys. 60, 537 (1988).

[37] F. Sciortino, P. Tartaglia, and E. Zaccarelli (2003), condmat/0304192.

[38] G. Foffi, W. Götze, F. Sciortino, P. Tartaglia, and T. Voigtmann (2003), preprint. 